\title{
Optimizing Nannochloropsis Growing Conditions for Biodiesel Production Through Analysis of Lipid Content
}

\author{
Steven D. Liu ${ }^{1}$, Devon Renock ${ }^{2}$
}

\begin{abstract}
Fossil fuels have propelled society to our current technology, but the future of energy lies in renewable resources, starting with vehicles. Despite constituting only $5 \%$ of the total vehicles in the United States, medium to heavy-duty trucks, which consume diesel fuel, are responsible for an astounding $23 \%$ of annual $\mathrm{CO}_{2}$ emissions in the transportation sector. The full implementation of greener biodiesel is often deemed an infeasible method for mitigating pollution because the production of such biodiesel directly competes with the agricultural industry for the available arable land. Energy-dense algae are more suitable feedstocks for biodiesel and circumvent many of the problems posed by current biodiesel feedstocks, and their potential can be used to propel the biodiesel industry into the future of sustainable energy. Nannochloropsis is a promising genus of algae due to its high productivity and lipid content. Here I show how to optimize the growing medium composition for increased biodiesel quality while maintaining high productivity by quantifying the constituent fatty acid type and composition using gas chromatography (GC). The algae are grown in two groups of three $2.5 \mathrm{~L}$ glass jugs spanning three concentration levels of nitrates and phosphates. A growing " $\mathrm{f} / 2$ " medium is kept constant across trials. The algae are harvested using a flocculating solution of aluminum sulfate and vacuum filtration. In situ transesterification is used to achieve maximum conversion of fatty acids into fatty acid methyl esters, which are then analyzed using GC. The algae grown in low, medium, and high nutrient concentrations produced average absorbance values (a measure of biomass concentration) at $750 \mathrm{~nm}$ of $0.91,0.99$, and 1.18 , respectively, after 32 days of growth. The maximum monounsaturated fatty acid (MUFA) concentration of $62.68 \%$ of total fatty acids was reached in a low nutrient concentration, which corresponds to high-quality biodiesel. Through this study, a scientific breakthrough was achieved by maximizing both the quality of biodiesel produced, which is beyond any currently available biodiesel, and also the quantity with a productivity of greater than 100 times the current biodiesel feedstocks.
\end{abstract}

\section{Keywords}

Algae; Energy; Biofuel; Biodiesel; Lipid Analysis; Nutrient Composition

${ }^{1}$ High School Junior at Shady Side Academy Senior School, Pittsburgh, Pennsylvania

${ }^{2}$ Science teacher at Shady Side Academy Senior School, Pittsburgh, Pennsylvania

\section{Contents}

1 Introduction

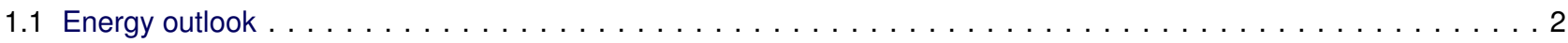

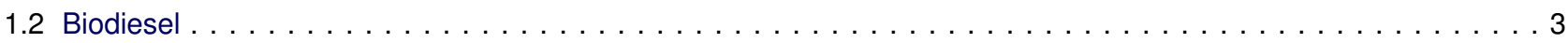

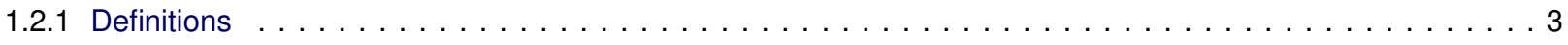

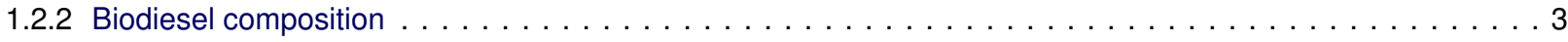

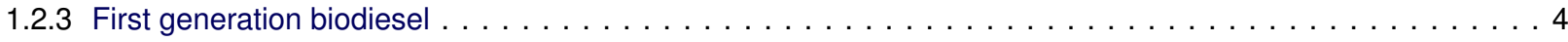

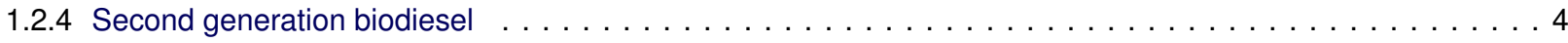

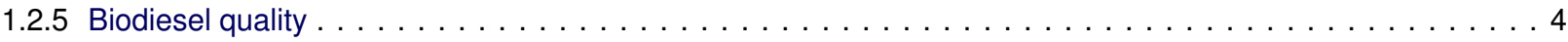

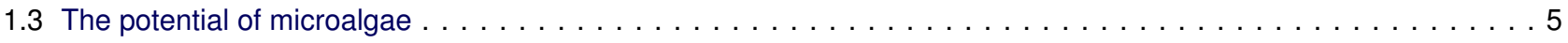

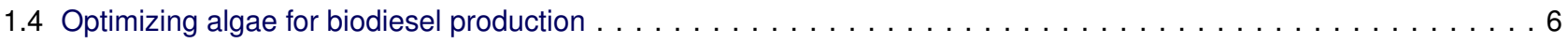

1.5 Theory supporting hypothesis: Lipid production $\ldots \ldots \ldots \ldots$

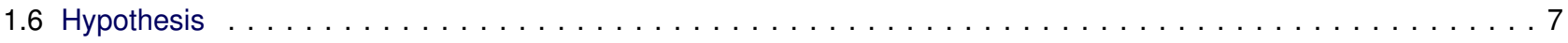

2 Methods $\quad 7$

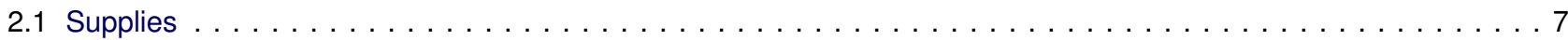

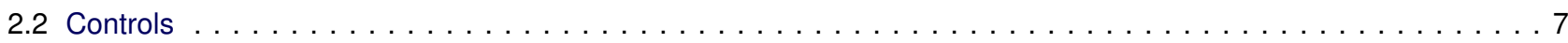

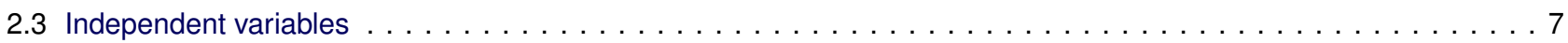




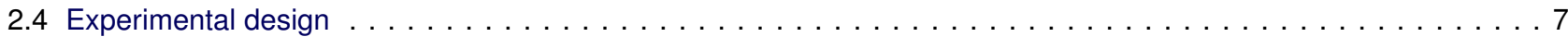

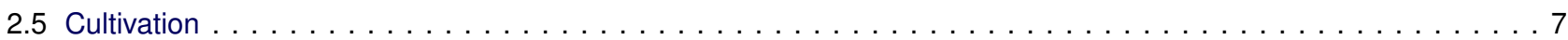

2.5.1 Cultivation of first non-experimental batch $\ldots \ldots \ldots \ldots \ldots$

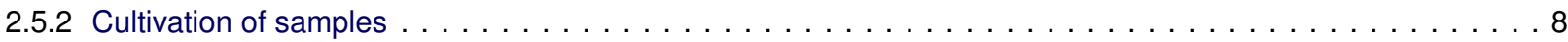

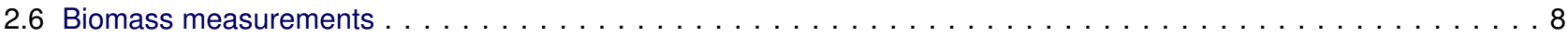

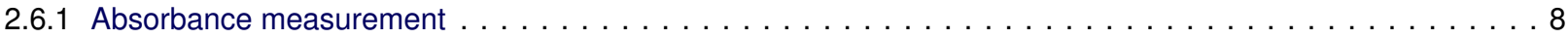

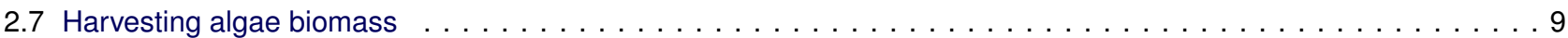

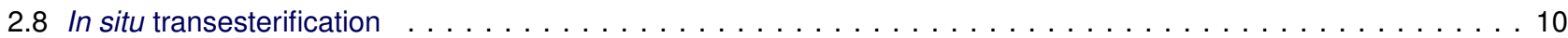

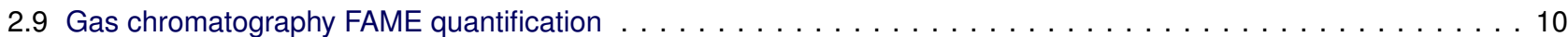

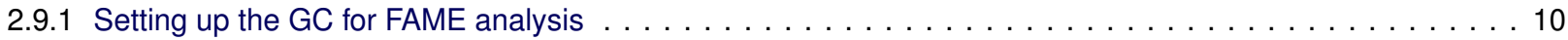

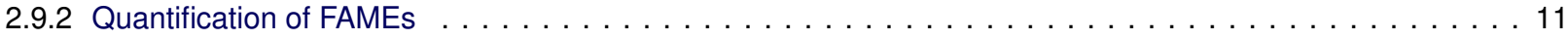

3 Results 11

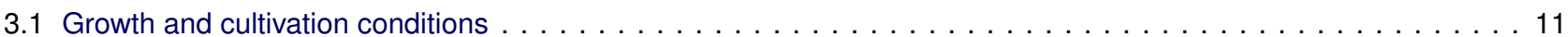

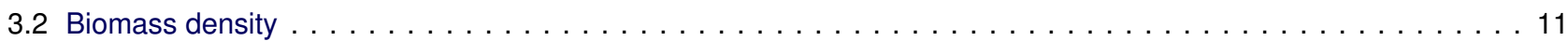

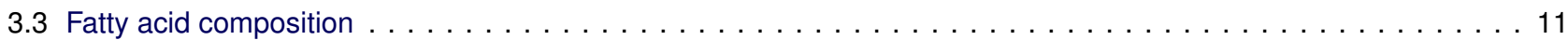

4 Possible Inaccuracies 13

4.1 Biomass density, growth, and cultivation conditions $\ldots \ldots \ldots \ldots \ldots$

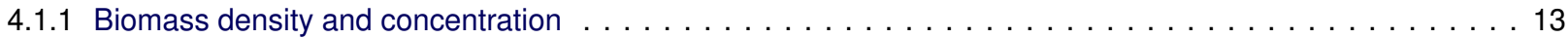

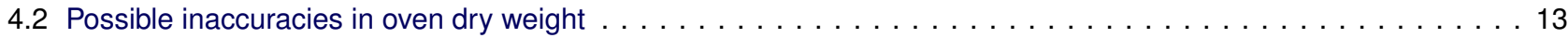

4.3 Possible gas chromatography analysis inaccuracies $\ldots \ldots \ldots \ldots \ldots$

5 Discussion 14

5.1 Nannochloropsis oculata produces increased relative MUFA concentrations in nutrient-stressed conditions . . . 14

5.2 Nannochloropsis oculata produces decreased total fatty acids in nutrient-stressed conditions . . . . . . . . . . 14

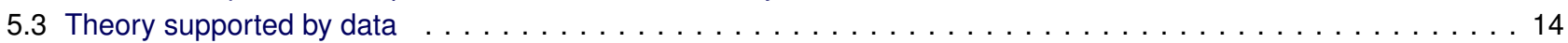

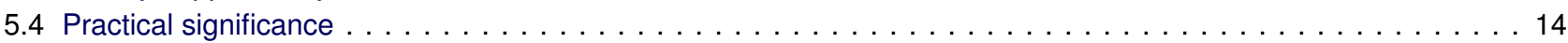

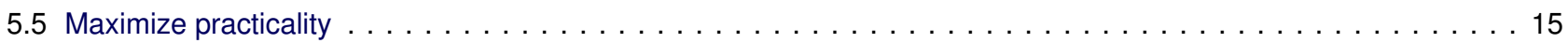

6 Conclusion 15

Acknowledgments 16

Appendices 17

References 19

\section{Introduction}

\subsection{Energy outlook}

In 2020, the annual world primary energy consumption, a measure of the total energy produced, was estimated at $5.8390 \times 10^{20}$ joules [1], a number with such a high order of magnitude that it is impossible to visualize. It is possible to visualize, though, that only a mere $10.4 \%$ of this energy was produced using renewable sources [1]. Although this seems to be a significant portion, Bill Gates recently stated in an interview with CBS that it is not nearly enough to prevent the devastating effects of climate change [2]. This increased threat of global climate change is mainly attributed to greenhouse gas (GHG) emissions from fossil fuel usage. The associated climatic change projections pose major consequences for nature and humans that will at best result in the doubling of atmospheric $\mathrm{CO}_{2}$ concentration compared to the previous naturally-occurring highest concentration of $300 \mathrm{ppm}$ within the next half-century $[3,4]$. Such predictions present a profound level of uncertainty regarding the environmental sustainability of current fossil fuel use not only about the re- source's finiteness but also concerning the negative effects of $\mathrm{CO}_{2}$ emissions.

Fossil fuels, as formally defined by U.S. Department of Energy, are non-renewable resources that are formed when prehistoric plants and animals die and are gradually buried by layers of rock [5]. Fossil fuels are the largest contributors of GHGs to the biosphere, totaling 34169.0 million tons of $\mathrm{CO}_{2}$ in 2020 [1], of which $28 \%$ is associated with the transportation sector [6]. This sector is the most polluting of all sectors, including industrial, agricultural, and electricity generation. The transportation sector produces over 55\% [7] of the nitrogen oxides (a much worse pollutant than $\mathrm{CO}_{2}$ with 265-298 times the global warming potential [8]) in the biosphere. The transportation sector is also the least renewable, with only 4\% [9] of its total energy derived from renewable sources, mainly biofuels. The worst offenders of this sector are medium to heavy-duty trucks, which represent 5\% [10] of the total vehicles in the US but account for an astounding 23\% [6] of annual $\mathrm{CO}_{2}$ and $96 \%$ of nitrogen oxides emissions [11].

Therefore, the overall implication is a need for an enhance- 
ment of renewable energy sources in the transportation sector. Such investment would produce the maximum return of reducing GHG emissions, especially in creating economical and practical biodiesel for medium to heavy-duty trucks.

\subsection{Biodiesel}

In recent years, the use of liquid biofuels in the transportation sector has shown rapid global growth. This is primarily motivated by: (1) GHG emissions and climate change concerns, (2) a desire for renewable and sustainable energy sources, and (3) an interest in developing more secure fuel supplies [12]. This growth is partially driven by policies focused on mitigating GHG emissions [1]. For example, the U.S. Energy Independence and Security Act (EISA) of 2007 requires the Environmental Protection Agency to set annual standards for the Renewable Fuel Standard program each year [13,14]. The annual biodiesel production requirement was set at 2.43 billion gallons/year for 2020 [14]; however, only 1.82 billion gallons were produced [15], resulting in fines for parties that failed to meet the requirement [16].

Although burning petroleum-based diesel and biodiesel both release greenhouse gases, the overall environmental impact of biodiesel is much lower. The combustion of petroleumbased diesel results in a net-positive greenhouse gas emission since it releases carbon originally captured and stored in the crust of Earth. This is harmful to the environment and causes global warming. However, since biodiesels are produced from biological feedstocks, it has a different mechanism of reaction. The feedstocks first capture carbon from the atmosphere through photosynthesis and store it in lipids. When the biodiesel produced from the feedstocks is burned in a diesel engine, the carbon is re-released into the atmosphere. Optimally, the process involving biodiesel is a net-zero carbon emission cycle. In reality, this is currently unattainable due to emissions during the processing of feedstocks and the production of biodiesel. Still, biodiesel produces much fewer greenhouse gas emissions than petroleum-based diesel.

\subsubsection{Definitions}

There are many types of diesel fuel that are marketed. The three categories are petroleum-based diesel, biodiesel, and renewable diesel.

Petroleum-based diesel (often referred to as "diesel") is a fossil fuel that is derived from petroleum. To produce diesel, petroleum is refined through a heat and pressure-based process called hydrogenation.

In contrast to non-renewable petroleum-based diesel, renewable diesel and biodiesel are both biomass-based biofuels and are therefore renewable fuels. However, renewable diesel and biodiesel differ in how they are produced and in their physical properties. Renewable diesel is processed similarly to the way petroleum-based diesel is produced, which makes it more chemically similar to diesel. Renewable diesel can be used in engines that are designed to run on conventional diesel fuel [17]. The significant disadvantage of renewable diesel is that the production process is highly inefficient and is not currently economically feasible other than in regions where there are significant government subsidies. In the United States, these subsidies are currently only present in the state of California [17].

Biodiesel is also a renewable fuel; however, it is produced differently than petroleum diesel and renewable diesel, using transesterification. Biodiesel is defined by ASTM International, formerly known as American Society for Testing and Materials, as "a fuel comprised of mono-alkyl esters of long-chain fatty acids derived from vegetable oils or animal fats, designated B100" [18]. Through the transesterification reaction, fatty acids are converted into fatty acid methyl esters (FAME) by exchanging the organic group $\mathrm{R}^{\prime}$ with the organic group $\mathrm{R}^{\prime \prime}$ of an alcohol, most commonly methanol in a biodiesel production reaction. FAMEs are more commonly known as biodiesel, and they are used directly in biodieselsuitable vehicles.

A simple fatty acid naming convention consists of two numbers separated by a colon symbol. The first and second numbers represent the number of carbon atoms and carboncarbon double bonds, respectively, in the fatty acid chain. The latter is more commonly known as the degree of unsaturation [12]. For example, C18:0 (octadecanoic acid) represents a fully saturated 18-carbon long fatty acid chain, which is a carboxyl group attached to a hydrocarbon chain. C18:1 represents a monounsaturated 18-carbon long fatty acid chain. $\mathrm{C} 18: 2, \mathrm{C} 18: 3$, and greater represent a polyunsaturated 18carbon long fatty acid chain. To indicate a fatty acid has been transesterified, "ME" is added to the abbreviation. For example, octadecanoic acid methyl ester (or methyl octadecanoate) is represented as C18:0ME. To signify when a particular unsaturated fatty acid is a cis- or trans-isomer, "-c" or "-t", respectively, is used as a subfix. For example, cis-octadecenoic acid is represented as $\mathrm{C} 18: 1 \mathrm{c}$.

\subsubsection{Biodiesel composition}

Biodiesel fuel is produced by transesterification of virtually any triglyceride feedstock. This includes transesterifing oil-producing crops, animal fats, and algal lipids following the reaction mechanism pictured in fig. 1. Biodiesel, which is composed of FAMEs, produced from transesterification of triglycerides are nearly exclusively composed of evennumbered fatty acid carbon-chains. In contrast, renewable diesel produced from the same feedstocks contain substantial amounts of odd-numbered fatty acid chains since one carbon is removed during the hydrotreating step [12]. This phenomenon is simply a difference in characteristics between biodiesel and renewable diesel and does not affect the quality of diesel produced. 


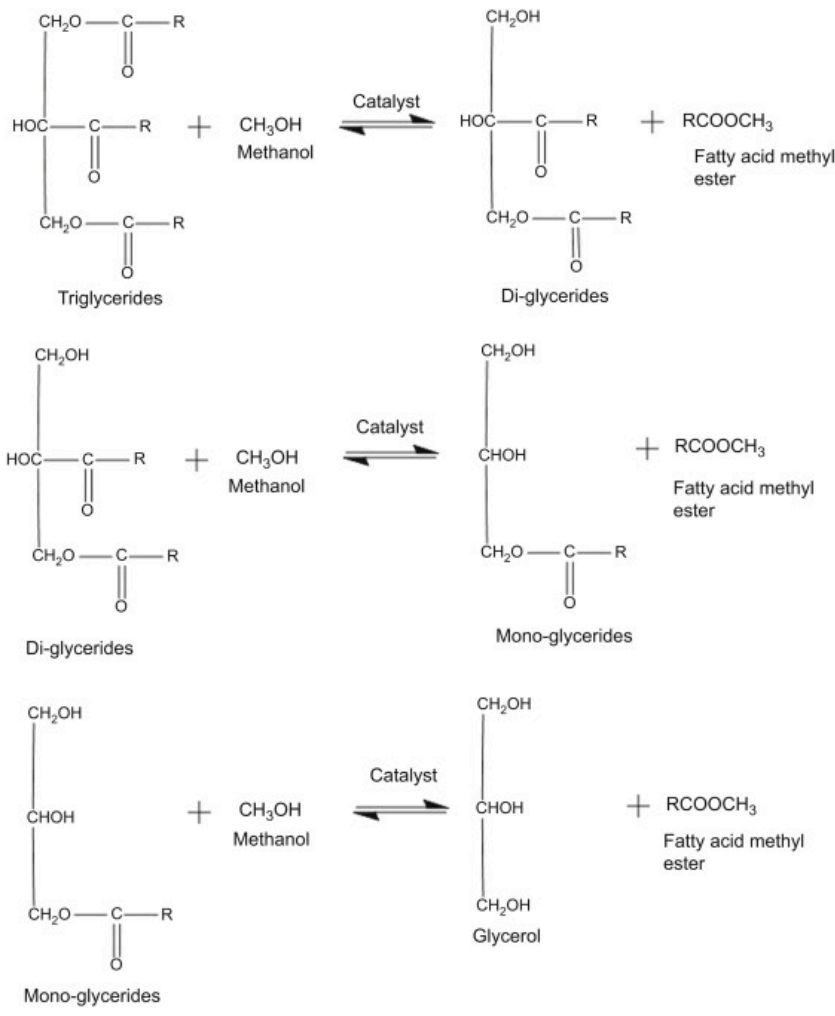

Figure 1. The transesterification mechanism of fatty acids [19].

\subsubsection{First generation biodiesel}

First-generation biodiesel that have attained economic production levels in small quantities are mainly extracted from food and oil crops, including rapeseed oil, sugarcane, sugar beet, and maize [20] as well as vegetable oils and animal fats using conventional technology [21]. The availability of crops and comparatively easy processing procedure are the main benefits of first-generation feedstocks. Although the growth in production and consumption of biodiesel will continue, their impacts towards meeting the overall energy demands in the transportation sector will become increasingly limited due to direct competition with food production for the use of arable land [22]. Therefore, first-generation biodiesel's exact benefits will soon become its limiting factor.

\subsubsection{Second generation biodiesel}

Second-generation feedstocks are produced from nonedible feedstocks, including Jatropha oil, Rubber seed oil, and Neem oil [23]. The main advantage of such feedstocks over first-generation feedstocks is little to no requirement of arable land. However, second-generation feedstocks are not currently used for biodiesel production in the U.S. [24] since they do not yield enough benefits, as of right now, compared to first-generation feedstocks to justify extensive research and development. Further, harvesting and treatment difficulties of second-generation feedstocks pose a significant challenge for economic production. Currently, demand for biodiesel can still be satisfied with first-generation feedstocks, although the limit is being reached quickly, which will require development of more practical feedstocks in the future [20].

\subsubsection{Biodiesel quality}

Another limiting factor on the wide adoption of biodiesel is the varying quality depending on production from various feedstocks, which affects the performance in many diesel engines. The fatty acids in the feedstocks are composed of different concentrations of monounsaturated fatty acids (MUFA), saturated fatty acids (SFA), and polyunsaturated fatty acids (PUFA). The quality of biodiesel and petroleum-based diesel can be measured by three numbers: (1) cetane number, (2) cloud point, and (3) oxidative stability. (1) The cetane number is a measure of the performance of a given diesel fuel in a diesel engine. A higher cetane number is desirable since it correlates with a higher performance fuel. (2) The cloud point, measured in degree Celsius, is a measure of the fluidity of diesel and biodiesel fuel at a low temperature; therefore, a lower number is desirable. (3) The oxidative stability is a measure of the storage capabilities of a diesel fuel. The measure of oxidative stability has units of milligrams of insoluble particles per 100 milliliters of fuel. Therefore, a lower number is desirable, which corresponds to a more pure fuel.

To explain why MUFA, SFA, and PUFA gives the fuel its characteristics, the molecular structure of these three molecules must be analyzed. SFA is a straight hydrocarbon chains with a carboxyl group attached. On the other hand, unsaturated fatty acids (MUFA and PUFA) are fatty acids with one or more bends, depending on the degree of desaturation. Pictured in fig. 2 are a straight-chain SFA, single-bend cisMUFA, and trans-MUFA. Trans-isomers are rarely found in biological organisms.

\section{Saturated fatty acid}

Stearic acid

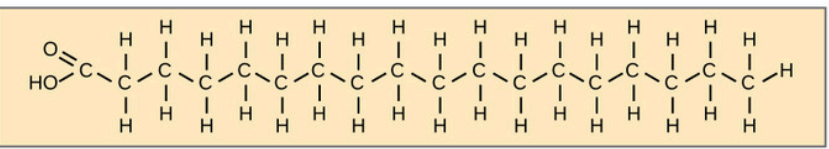

Unsaturated fatty acids

Cis oleic acid

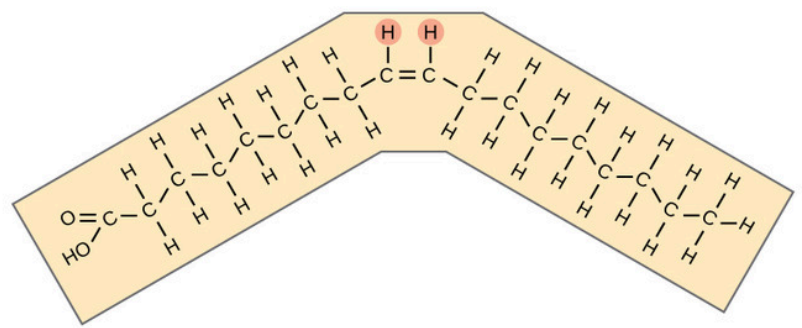

Trans oleic acid

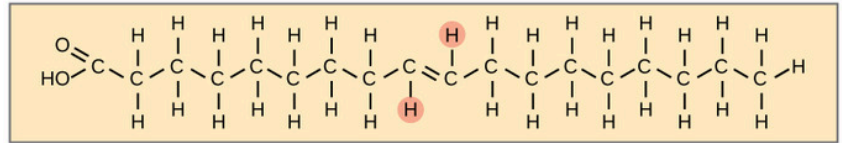

Figure 2. Pictured are the molecular structures of SFA and cis- and trans-MUFA [25]. 


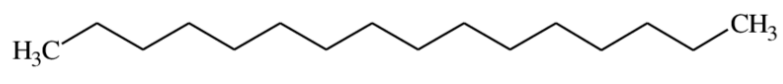

n-Hexadecane (cetane), cetane number $=100$<smiles>Cc1ccc2ccccc2c1</smiles>

1-Methylnaphthalene, cetane number $=0$

Figure 3. Pictured are the molecular structures of n-hexadecane (cetane) and 1-methylnaphthalene [26].

The cetane number compares the molecular structure of a molecule to n-hexadecane [27], which is more commonly known as cetane (fig. 3). The straight-chain hydrocarbon n-hexadecane is defined to have a cetane number of 100. 1Methylnaphthalene is defined to have a cetane number of 0 . SFA has the most similar structure to n-hexadecane, so it has a higher cetane number. MUFA has one bend, thus a lower cetane number. PUFA has multiple bends, thus the lowest cetane number of the three types of fatty acids.

In SFAs, the straight-chain structure allows molecules to be packed closely together [28]. This results in the biodiesel that is derived from SFA to turn solid at a higher temperature (a higher cloud point) than biodiesel derived from unsaturated fatty acids. On the other hand, as the degree of unsaturation increases, more bends are introduced into the molecules, which causes them to be less compact. This results in PUFA-derived biodiesel having the lowest cloud point.

It must be noted that the desaturation of fatty acids is the removal of hydrogen atoms that are bonded to the carbonchain. If hydrogen atoms are removed, the carbon atoms become more easily accessible by oxygen and are oxidized more quickly [29]. Therefore, biodiesel derived from fatty acids with more unsaturation will have a lower oxidative stability than that of the biodiesel derived from fatty acids with less unsaturation.

According to the US Department of Energy, the optimal biodiesel is produced only using MUFAs and no SFAs or PUFAs [18]. As shown in table 1, SFA-derived biodiesel has a high cetane number and oxidative stability, both desirable attributes, but has a high cloud point, which is undesirable since the biodiesel would become cloudy at a higher temperature than optimal. On the other hand, PUFA-derived biodiesel has a low cloud point, which is desirable, but has a low cetane number and oxidative stability, both less than optimal. A high MUFA content represents a compromise between the advantages and disadvantages of PUFAs and SFAs. It has an adequate cold-temperature fluidity, oxidative stability, and high cetane number [28]. However, no biological organism can produce only one type of fatty acids. The fatty acid profile is always a combination of the three types [18]. This is a significant challenge since any SFA and PUFA would decrease the quality of the biodiesel produced.

Currently, the main biodiesel feedstock in the U.S. is soybean oil, the precursor to $57 \%$ of the biodiesel produced [30].
Soybean oil is widely adopted due to its low demand in other sectors and, therefore, increased availability. However, soybean oil is composed of $23.7 \%$ MUFA [12], much less than desirable, producing a lower quality biodiesel.

On the other hand, a small portion of biodiesel, $10 \%$, is produced from canola oil [30], which has the highest quality with 62.9\% MUFA [12]. Although canola oil-based biodiesel has a higher quality, it cannot be widely adopted due to high demand as a cooking oil.

In summary, the broad range of biodiesel quality and uncertainty about the quality of biodiesel that one might be purchasing greatly limits the practicality of biodiesel since not all diesel vehicles can use biodiesel. Biodiesel of lower quality is not even considered as an option. Although canola oil produces high-quality biodiesel, its use is limited. This presents the difficulty of the biodiesel industry competing with the agricultural industry, thus, a different feedstock is needed to mitigate these difficulties.

\subsection{The potential of microalgae}

Usage of first-generation feedstock for biodiesel production is gradually approaching its limits due to increasing food prices and occupation of arable land [22]. Second-generation feedstocks solve some of these problems but create other problems during the production of biodiesel, as outlined in section 1.2.4 [20]. Thus, a new and innovative energy source is required to mitigate the disadvantages of first and secondgeneration feedstocks.

Microalgae (more commonly referred to as algae) is considered a third-generation feedstock for biofuels. An alternative for first and second-generation feedstocks, thirdgeneration algae feedstocks have significant benefits. The major benefits of third-generation biodiesel are: (1) lesser greenhouse effect, (2) higher productivity and more easily cultivated and harvested [31-33], (3) decreased requirement of arable land (therefore eliminating competition with the agricultural industry), (4) higher lipid concentration than traditional feedstocks, and (5) decreased influence on food supply [23].

As stated previously, the most optimal solution to the current climate crisis is to create widely available and practical biodiesel, which is more than possible with algae due to their high lipid content of 20\%-50\% dry biomass [34]. Not only this, but algae can also be effectively used for ethanol production due to their cellulosic structure containing high carbohydrates. The remaining carbohydrates after lipid extraction can be used to produce ethanol, thus creating two products using one feedstock. Therefore, using algae not only provides a scientific solution to have both high quality and high productivity while maintaining practicality, but also provides economic benefits for the society and industry by producing useful byproducts without a need for further dedicated energy during the cultivation process.

The most significant advantage of using microalgae is its low land requirement. Algal production areas do not occupy 


\begin{tabular}{l|lll}
\hline & Saturated (SFA) & Monounsaturated (MUFA) & Polyunsaturated (PUFA) \\
\hline Fatty acid & C12:0, 14:0, 16:0, & C16:1, 18:1, 20:1, & C18:2, 18:3, 20:4, \\
& $18: 0,20: 0,22: 0$ & $22: 1$ & $20: 5$ \\
\hline Cetane Number & High & Medium & Low \\
Cloud Point & High & Medium & Low \\
Oxidative Stability & High & Medium & Low \\
\hline
\end{tabular}

Table 1. Fuel Properties as a Function of Fuel Composition in Diesel Engines [18].

agricultural land, and they only require fertilizer to control the quality of algae produced in terms of fatty acid types. Further, algal feedstocks maintain a very high productivity of over 100 times of existing feedstocks (table 2) while eliminating competition with the agricultural sector.

\begin{tabular}{l|c}
\hline Feedstock & $\begin{array}{c}\text { Biodiesel productivity } \\
\text { kg biodiesel/ha/year }\end{array}$ \\
\hline Algae & 121,104 \\
Canola oil & 862 \\
Soybean oil & 562 \\
\hline
\end{tabular}

Table 2. Biodiesel yield per unit area per year for various feedstocks [35].

Possible algal production areas include factory grounds and sewage treatment plants. The rich nutrients in wastewater can be used to boost algal growth while simultaneously filtering the wastewater, removing the need for large dedicated water treatment plants through chemical processes. Algal growth can also be enhanced using $\mathrm{CO}_{2}$ [36] from industrial sources such as fossil fuel-fired power plants and direct-air capture plants designed to capture $\mathrm{CO}_{2}$ from the air. In this way, algae may be considered carbon-neutral and only reusing existing $\mathrm{CO}_{2}$ in the atmosphere. Therefore, using biodiesel will have a minimal impact on the environment due to the carbon-neutrality of the algae used to produce biodiesel. By reducing the competition for arable land and food sources, algae allow for further expansion of the biofuel industry while maintaining a carbon-neutral product.

\subsection{Optimizing algae for biodiesel production}

As previously stated, the greatest return on investment will be deriving biodiesel using algal lipids. Currently, biodiesel produced by algae is of lower quality than that which is produced by vegetable oils, which are the primary feedstocks used for biodiesel production in the U.S. [30] This is mostly due to the composition of the fatty acids produced by the algae, which are often slightly lower in MUFAs and higher in SFAs and PUFAs compared to vegetable oils. As previously stated, the optimal biodiesel is produced using only MUFAs.

It is crucial that a high-quality biodiesel comparable to petroleum-based diesel is produced since this would maximize the practicality and effectiveness of the biodiesel. Having large amounts of low-quality fuel is not practical since it cannot be used in many engines. On the other hand, producing slightly smaller amounts of high-quality fuel maximizes its practicality since more vehicles can use the fuel. Thus, it is vital for the industry and environment to formulate a highquality biodiesel feedstock that is high in MUFA and low in SFA and PUFA.

This research study is focused on formulating the optimal growing medium concentration of various nutrients to maximize the relative MUFA content of the algal lipids while minimizing PUFA and SFA (see section 1.5 for further explanation). The goal is to optimize the relative MUFA content beyond that of canola oil to produce the highest quality biodiesel comparable to petroleum-derived diesel.

Past research has shown that the genus of algae Nannochloropsis has one of the highest overall lipid contents [37]. Further, it is easily cultivated, partially due to its inherent charge that causes individual alga to repel each other and maintain the algae in suspension [38]. Therefore, Nannochloropsis is chosen to be the research species in this study since it is the optimal genus for biodiesel production. The species obtained is Nannochloropsis oculata.

\subsection{Theory supporting hypothesis: Lipid produc- tion}

The triglycerides used to produce biodiesel are composed of three fatty acids and a glycerol. The molecules of interest are fatty acids. The formation of fatty acids involving fatty acid synthase occurs via six recurring reactions until the 16carbon chain palmitic acid is produced (C16:0) [39]. Once this fatty acid has been produced, it can undergo a number of modifications that result in desaturation from the enzyme desaturase or elongation from elongase. These two modifications often correlate with each other. Meaning, a greater degree of overall desaturation among all fatty acids is often associated with greater overall elongation. This does not mean that a specific fatty acid that is less saturated is also longer; instead, this mechanism regards a common trend in the entire group of fatty acid molecules in general.

The reason that a higher lipid concentration would result in a greater overall desaturation is as follows. In a higher lipid concentration, the algae would have more energy to modify the C16:0 fatty acid more, thus resulting in an overall increase in desaturation and more PUFAs. On the other hand, in a lower lipid concentration, the algae would have less energy and fewer fatty acids to desaturate. Therefore, more fatty acids are desaturated only once, resulting in a relative increase 
in concentration of MUFAs.

\subsection{Hypothesis}

Previous research has shown that the concentration of lipids in algal biomass increases as the concentration of nitrates and phosphates in the media that the algae is grown in increases [34]. As the lipid concentration increases, the concentration of longer chain fatty acids also increases [37], which is associated with an increase in desaturation, as previously stated. In this study, it is hypothesized that the contrary is also true: a lower concentration of nitrates and phosphates should result in relatively less desaturation of fatty acids. Therefore more lipids would have gone through only one stage of desaturation, producing a relative increase in MUFA concentration.

I hypothesize that the Nannochloropsis grown in low nitrate and low phosphate concentrations would yield a higher MUFA percentage and lower SFA and PUFA percentage, which would produce higher quality biodiesel, at the expense of a slight decrease in productivity. The reason for a slight decrease in productivity is hypothesized to be that the algae would produce a decreased biomass concentration due to the fewer nutrients present and result in a relatively lower lipid concentration.

\section{Methods}

\subsection{Supplies}

The instrumentation used in this study included: gas chromatography (GC) with flame ionization detector, UVvis spectrometer, standard lab equipment (beakers, flasks, graduated cylinders, etc.), Nannochloropsis oculata (from www.algaeresearchsupply.com), 2.5L glass jugs for algae growing, grow lights, and air pump.

\subsection{Controls}

The following variables will be held constant across the six growing containers:

- Algae grown: Nannochloropsis oculata

- The same algae is grown across all experimental growing mediums.

- Culture medium

- The same culture medium composition (Guillard (1975), [40]) is used across all experiments EXCEPT for the nutrients specified in section 2.3.

- Light illuminance: 30-35 lux

- Held constant using fluorescent grow light in a dark room kept at a consistent distance from the grow containers. Illuminance is measured using a Vernier light probe (LS-BTA).

- It has been shown that algae produces the highest concentration of lipids (including MUFA, PUFA, and SFA based lipids) at this illumination.

- Temperature: $22.5-23.5^{\circ} \mathrm{C}$

- A heating mat is placed under algae growing containers. Power delivered to the heating mat is varied automatically based on temperature.

- $\mathrm{pH}$ : 9.63-9.91

- Buffering salts are added to the artificial seawater medium.

- Growth volume: $2 \mathrm{~L}$

- Water is added as needed to each $2 \mathrm{~L}$ growing algae suspension in $2.5 \mathrm{~L}$ glass jugs to mitigate the effects of evaporation.

\subsection{Independent variables}

The algae is grown in the Guillard (1975) " $\mathrm{f} / 2$ " Medium [40]. The only modifications made to the medium are the concentrations of $\mathrm{NaNO}_{3}$ and $\mathrm{NaH}_{2} \mathrm{PO}_{4} \cdot 2 \mathrm{H}_{2} \mathrm{O}$ :

- Low NP (Nitrate and Phosphate) - 1/2 the concentration of baseline

- $\mathrm{NaNO}_{3}: 4.41 \times 10^{-2} \mathrm{M}$

- $\mathrm{NaH}_{2} \mathrm{PO}_{4} \cdot 2 \mathrm{H}_{2} \mathrm{O}: 1.81 \times 10^{-5} \mathrm{M}$

- Baseline (Original $\mathrm{f} / 2$ Medium)

- $\mathrm{NaNO}_{3}: 8.82 \times 10^{-2} \mathrm{M}$

- $\mathrm{NaH}_{2} \mathrm{PO}_{4} \cdot 2 \mathrm{H}_{2} \mathrm{O}: 3.62 \times 10^{-5} \mathrm{M}$

- High NP - 2 times the concentration of baseline

$-\mathrm{NaNO}_{3}: 1.76 \times 10^{-1} \mathrm{M}$

$-\mathrm{NaH}_{2} \mathrm{PO}_{4} \cdot 2 \mathrm{H}_{2} \mathrm{O}: 7.24 \times 10^{-5} \mathrm{M}$

\subsection{Experimental design}

Nannochloropsis oculata was cultivated for 32 days at three different levels of nutrients as specified in section 2.3. The baseline nitrate and phosphate concentration used is the same as specified by the $\mathrm{f} / 2$ medium. The high and low were chosen to double and halve the concentrations, respectively. The $\mathrm{f} / 2$ medium, which is my baseline, is derived from the original $\mathrm{f}$ medium, which had concentrations at twice the concentration of $f / 2$, hence the name $f$ divided by 2 . To test the $f$ medium condition, the high nutrient concentration was chosen to be the same as the f medium, which is twice the concentration in the $\mathrm{f} / 2$ medium. The low nutrient concentration was chosen to halve the $f / 2$ concentration to maintain symmetry.

Each cultivation was performed in duplicate to increase statistical validity, completing a $2 \times 3$ design. Throughout this study, each sample is labeled as L1, B1, H1 for the first group of low, baseline, and high nutrient concentrations, respectively. The second group is labeled as L2, B2, H2 for the second group of L-NP, Baseline, and H-NP. Due to space constraints, cultivations were performed in duplicate. Cultivations were conducted in a temperature and light-controlled room using six glass jugs.

Each analyte produced from individual algae suspension samples is analyzed in triplicate using GC to increase statistical validity.

\subsection{Cultivation}

The green microalgae Nannochloropsis oculata (obtained from Algae Research and Supply) is cultivated in six individual containers. The algae are grown on a 20 " x 48 " $100 \mathrm{~W}$ heating mat to maintain a constant temperature of $25^{\circ} \mathrm{C}$ [38]. 
A fluorescent grow light set on a 12:12 hour light:dark photoperiod is used to keep the illuminance at 30-35 lux. Six 1.3 L/min air stones are connected to a $58 \mathrm{~W}, 75 \mathrm{~L} / \mathrm{min}$ air pump to aerate the algae at a constant rate. A dissolved oxygen probe is not available, so an oxidation-reduction potential probe is used to measure the dissolved oxygen concentration since the reading is directly proportional to the amount of dissolved oxygen. See the configuration of full cultivation apparatus (fig. 4).

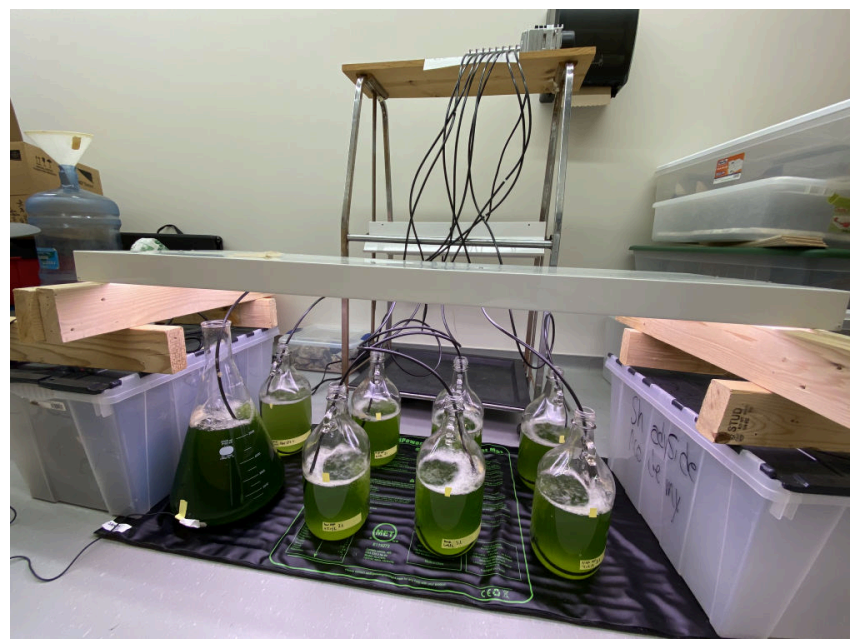

Figure 4. All batches of algae. Pictured is the full cultivation apparatus.

\subsubsection{Cultivation of first non-experimental batch}

A $50 \mathrm{~mL}$ inoculum of the algae, salts, and $\mathrm{f} / 2$ nutrients were obtained. The salts and $\mathrm{f} / 2$ nutrients were dissolved in $3.79 \mathrm{~L}$ of deionized water to create the $\mathrm{f} / 2 \mathrm{2}$ Medium. The first batch of algae will not be used to collect data. Instead, it will be grown in the baseline $\mathrm{f} / 2$ growing medium and will be used to inoculate the experiments. To grow the first batch of algae, the solution is placed in a 6 L Erlenmeyer flask, and the water level is recorded using tape on the side of the flask. The original absorbance of the $50 \mathrm{~mL}$ inoculum, which is directly proportional to the biomass concentration, is taken at $750 \mathrm{~nm}$ using a UV-vis spectrometer, which is the recommended wavelength to use [38] due to a non-linear increase in chlorophyll density as biomass density increases. The growing medium is inoculated using the $50 \mathrm{~mL}$ inoculum. Over several weeks, the algae will grow in the cultivation medium (fig. 5). When the absorbance of the algae suspension meets or exceeds the original value, proceed to the next step of inoculating the experimental flasks with the non-experimental algae suspension (section 2.5.2).

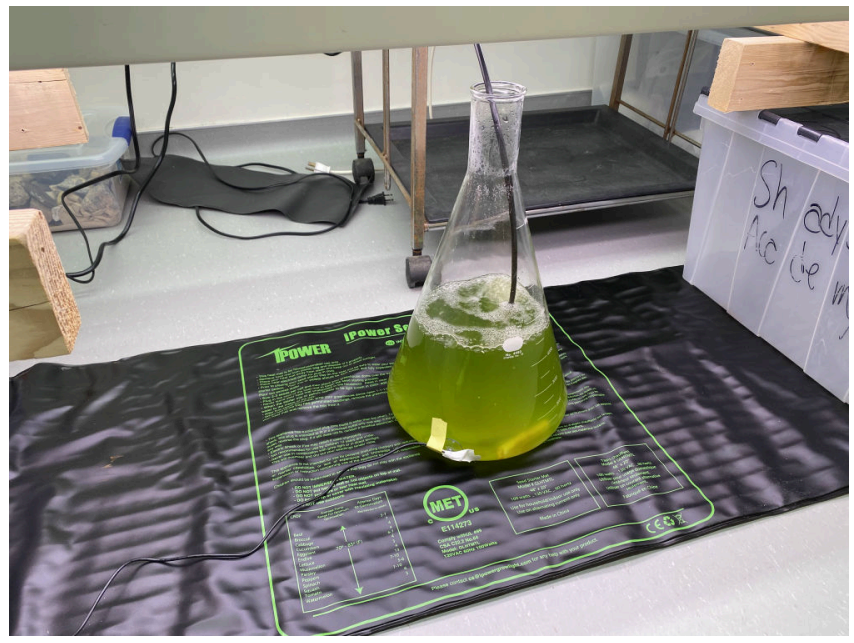

Figure 5. Non-experimental algae to be used to inoculate sample media. Pictured is the fluorescent grow light, heating mat, and air bubbler.

\subsubsection{Cultivation of samples}

Prepare duplicates of the three $2 \mathrm{~L} \mathrm{f} / 2$ Medium in a $2 \mathrm{~L}$ volumetric flask following the specified $\mathrm{NaNO}_{3}$ and $\mathrm{NaH}_{2} \mathrm{PO}_{4}$ - $2 \mathrm{H}_{2} \mathrm{O}$ concentrations (section 2.3). Transfer the medium to $2.5 \mathrm{~L}$ glass jugs. Inoculate each jug with $50 \mathrm{~mL}$ of algae suspension taken from the first batch of algae. Over several weeks, the algae will grow in the cultivation medium (fig. 6). When the absorbance of the algae suspension meets or exceeds the original value, proceed to the next step of harvesting algal biomass (section 2.7).

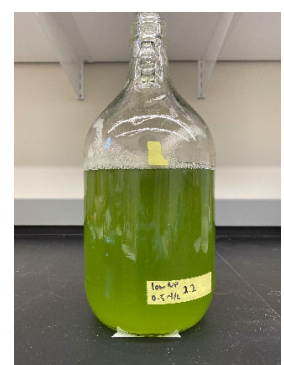

(a) L1 Algae Suspension

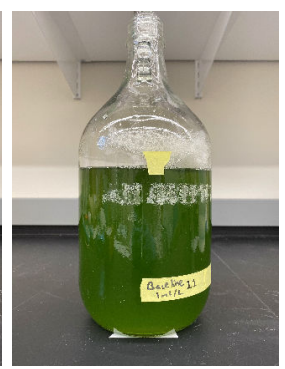

(b) B1 Algae Suspension

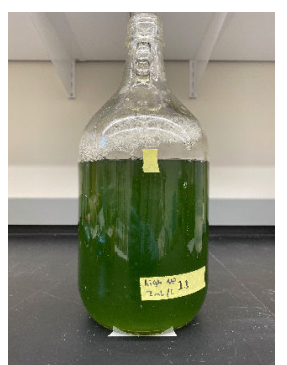

(c) H1 Algae Suspension
Figure 6. Algae growing in suspension in $2.5 \mathrm{~L}$ glass jug with $2 \mathrm{~L}$ growing media at different nutrient concentrations

\subsection{Biomass measurements}

\subsubsection{Absorbance measurement}

Samples of algae suspension at each nutrient level are collected in separate micro-cuvettes. A UV-vis spectrometer is used to measure the absorbance at $750 \mathrm{~nm}$ of the algae suspension. The absorbance is a measure of how much a substance, in this study the algae suspension, is able to absorb light at a specific wavelength, which is chosen to be $750 \mathrm{~nm}$. This wavelength is chosen due to the non-linear chlorophyll accumulating characteristic of algae as cell density increases. Choosing 
a wavelength out of the range of chlorophyll allows the spectrometer to minimize interference [38]. The absorbance value produced is directly proportional to the biomass concentration of algae biomass in the growing medium [41].

Once harvested, the precise biomass can be calculated based on the volume of suspension harvested and the mass of algae harvested (see section 2.7).

\subsection{Harvesting algae biomass}

The algae are harvested from suspension using the process of flocculation, which uses a charged flocculant that interacts with the algae's surface charge to produce flocs much greater in size than algal cell size.

A stock solution of $20 \mathrm{~g} / \mathrm{L} \mathrm{Al}_{2}\left(\mathrm{SO}_{4}\right)_{3}$ is prepared in deionized water and stored at room temperature. The precipitant is allowed to settle, and the transparent solution is used. Six beakers ( $250 \mathrm{~mL}$ each with $200 \mathrm{~mL}$ of algae suspension) of algae suspension is harvested from each experimental group and placed on a stir-plate (fig. 7). The flocculation test methodology is adapted from Chatsungnoen et al. [41]. All beakers were simultaneously treated as follows: (1) $6 \mathrm{~mL}$ of $20 \mathrm{~g} / \mathrm{L}$ $\mathrm{Al}_{2}\left(\mathrm{SO}_{4}\right)_{3}$ added using micropipette, (2) rapid mixing at 80 rpm for 2 min to disperse flocculant, (3) gentle mixing at 50 rpm to allow flocculation, and (4) no agitation for $30 \mathrm{mins}$ to allow flocs to settle (see fig. 8 and fig. 9).

The resulting flocs are harvested using vacuum-filtration with Whatman Grade 42 filter paper with a pore size of $2.5 \mu \mathrm{m}$ (fig. 10). Each sample is placed in a sterile petri dish (fig. 11) in a vacuum desiccator (not pictured). Samples placed under vacuum in a convection oven at $40^{\circ} \mathrm{C}$ for 12 hours to determine the weight of total dry biomass harvested.

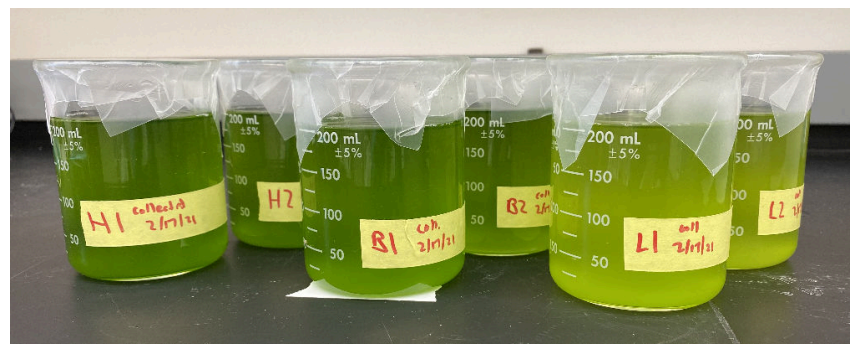

Figure 7. All samples are harvested. $200 \mathrm{~mL}$ of algae suspension placed in $250 \mathrm{~mL}$ beakers.

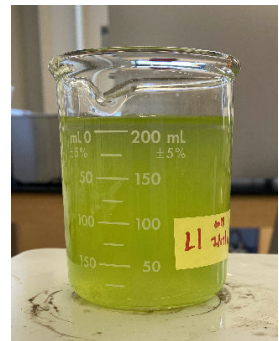

(a) L1 Sample

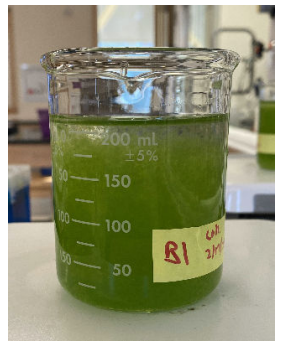

(b) B1 Sample

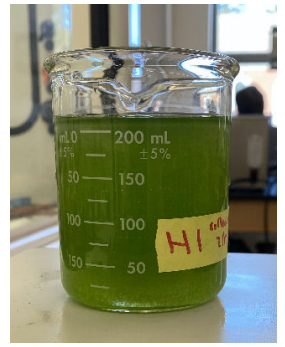

(c) H1 Sample
Figure 8. Flocculation of samples using $\mathrm{Al}_{2}\left(\mathrm{SO}_{4}\right)_{3}$. Image taken during flocculation period. Flocs are visible and are beginning to settle.

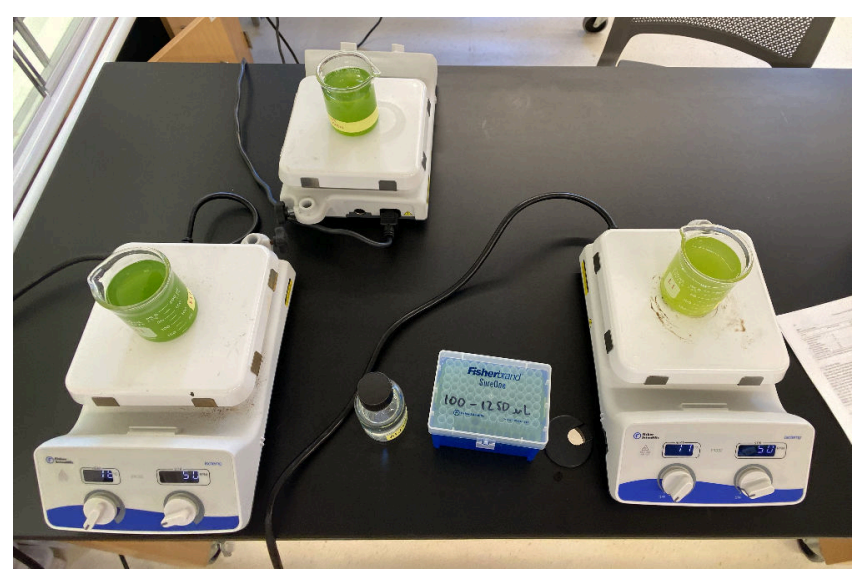

Figure 9. Flocculation of samples using $\mathrm{Al}_{2}\left(\mathrm{SO}_{4}\right)_{3}$ while being stirred using stir plate.

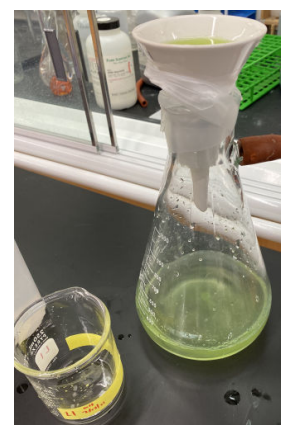

(a) L1 Filtration

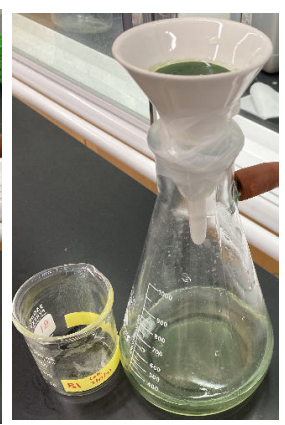

(b) B1 Filtration

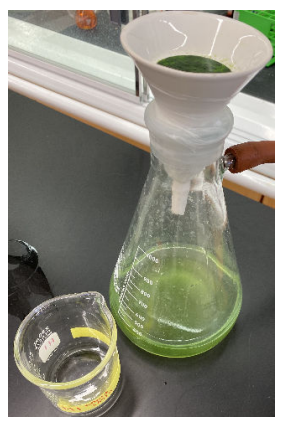

(c) H1 Filtration
Figure 10. Vacuum filtration of samples that have been flocculated. 


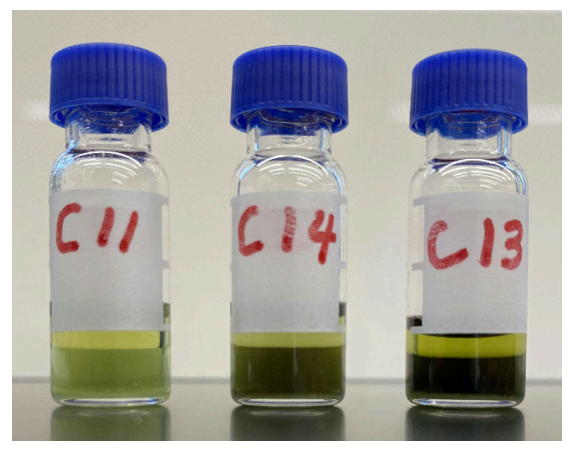

(a) Transesterified samples stored in $1.5 \mathrm{~mL}$ GC vials. Pictured are samples after performing hexane extraction, which produced transparent upper layer of hexane. The translucent bottom layer contains a mixture of FAMEs and glycerol. C11-L1 Sample; C14-B1; C13-H1.

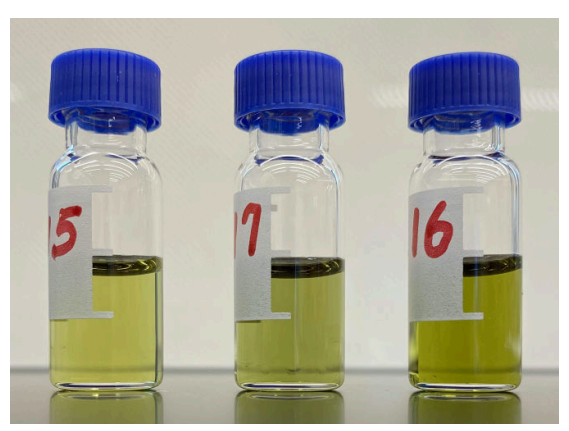

(b) Transparent upper layer of hexane after performing hexane extraction. Transferred to separate $1.5 \mathrm{~mL} \mathrm{GC}$ vials for storage. C15-L1 Upper hexane layer; C17-B1; C16-H1.

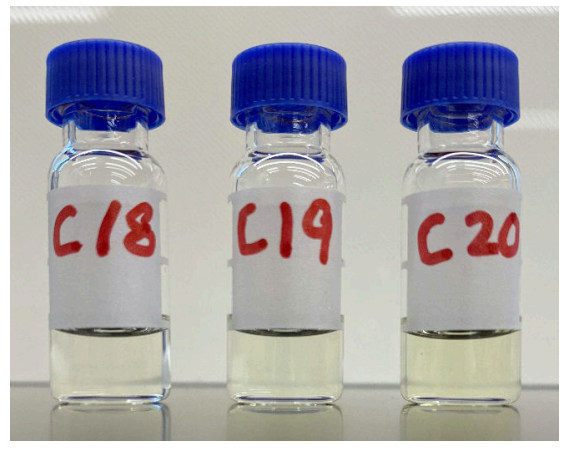

(c) Diluted upper hexane layer 2:3 (solution:hexane). Pictured is a dilution ratio of 1:9, which was replaced with 2:3 later during GC analysis since a ratio of 1:9 failed to produce quantifiable results due to low concentration.

C18-L1 hexane dilution; C19-B1; C20-H1.

Figure 12. Samples are transesterified, FAMEs extracted, and diluted for GC analysis.

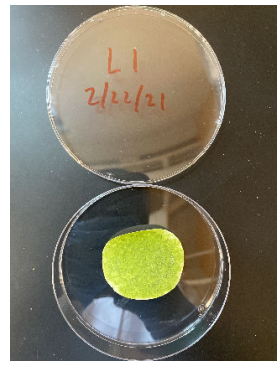

(a) L1 Sample

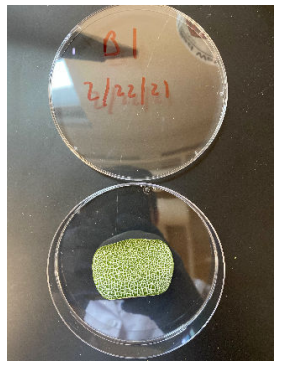

(b) B1 Sample

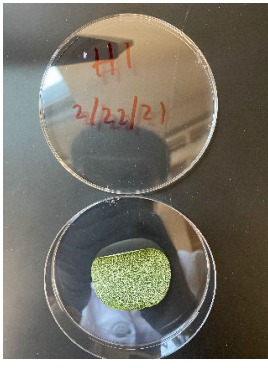

(c) H1 Sample
Figure 11. Samples have been dried and are stored in sterile petri dishes.

\subsection{In situ transesterification}

To produce biodiesel, algal lipids must be extracted from harvested biomass and transesterified into FAMEs. A singlestep in situ transesterification eliminates the need for an initial lipid extraction through direct transesterification of lipids performed simultaneously with lipid extraction. In situ transesterification also offers the advantage of quantifying all fatty acids as FAMEs, regardless of lipid extraction efficiency [42]. This allows an accurate representation of biodiesel potential.

It is possible to identify lipid composition without transesterification of its constituent fatty acids since each fatty acid directly correlates to one FAME. However, quantification of such fatty acids through GC is challenging due to the high elution temperatures of fatty acids. On the other hand, FAMEs have a much lower elution temperature, which is much more suitable for GC quantification [43].

The in situ transesterification methodology is adapted from Wychen et al. [44]. The method is carried out on 5 to $10 \mathrm{mg}$ of dried sample, extracting lipids in $0.2 \mathrm{~mL}$ chloroform:methanol $(2: 1, v / v)$, and simultaneously tranesterifying the lipids in situ with an acid-catalyzed reaction using $0.3 \mathrm{~mL}$ $\mathrm{HCl}$ :methanol $\left(0.6 \mathrm{M} \mathrm{HCl}\right.$ in methanol) for $1 \mathrm{hr}$ at $85^{\circ} \mathrm{C}$ in the presence of $250 \mu \mathrm{g}$ pentadecanoic acid methyl ester (or methyl pentadecanoate, C15:0ME) as an internal standard (fig. 12a). The resulting FAMEs are extracted with $1 \mathrm{~mL}$ HPLC grade hexane at room temperature for at least $1 \mathrm{hr}$ (fig. 12b), leaving behind polar compounds, e.g. glycerol or phosphatidic acid. The resulting FAME solution in hexane is diluted 2:3 (solution:hexane) with hexane (fig. 12c).

All extraction and transesterification procedures are performed in $1.5 \mathrm{~mL}$ glass $\mathrm{GC}$ vials.

\subsection{Gas chromatography FAME quantification}

The final hexane dilution from section 2.8 is analyzed using GC to quantify the FAME and therefore fatty acid composition. Supelco 37-component standards were used for identification and quantification of FAMEs with five-point calibrations ( 0 to $10 \mathrm{mg} / \mathrm{mL}$ ). Transesterification adds a methyl group to a fatty acid; thus, GC analysis of FAMEs overestimates the mass of fatty acids per unit biomass [45]. To mitigate this, data was mass-corrected so that results produced are in terms of $\mathrm{mg}$ of fatty acid per gram dry biomass.

\subsubsection{Setting up the GC for FAME analysis}

FAMEs were separated and quantified using a Thermo Scientific Trace 1310 GC equipped with a flame ionization detector (FID), split injector, and a TG-5MS $30 \mathrm{~m}$ x $0.25 \mathrm{~mm}$ inner diameter $x 0.25 \mu \mathrm{m}$ film thickness column. The analysis program that was developed is detailed below:

- $1 \mu \mathrm{L}$ injection at 50:1 split ratio, inlet temperature of $230^{\circ} \mathrm{C}$

- Constant flow: $1 \mathrm{~mL} / \mathrm{min}$ helium

- Oven temperature: $130^{\circ} \mathrm{C}$ for $1 \mathrm{~min}, 6^{\circ} \mathrm{C} / \mathrm{min}$ up to $170^{\circ} \mathrm{C}$ and hold for $18 \mathrm{~min}, 6^{\circ} \mathrm{C} / \mathrm{min}$ up to $213^{\circ} \mathrm{C}$, $8^{\circ} \mathrm{C} / \mathrm{min}$ up to $250^{\circ} \mathrm{C}$ and hold for $12 \mathrm{~min}(49.46 \mathrm{~min}$ total)

- FID: $250^{\circ} \mathrm{C}, 350 \mathrm{~mL} / \mathrm{min}$ zero air, $35 \mathrm{~mL} / \mathrm{min}_{2}$ 


\subsubsection{Quantification of FAMEs}

An internal standard method was chosen to account for possible losses during the transesterification process and other preparation processes.

The Flame Ionization Detector (FID) uses a flame fueled by zero air (ultra-pure air) and hydrogen. After ionizing the molecules, the FID measures the current produced in picoamperes (pA). This characteristic causes the FID to have a different response for different molecules. The response of all hydrocarbons is generally considered to be roughly proportional to their molecular weight [46]. To account for this, response factors were calculated for each fatty acid analyzed from analyzing the Supelco 37-component FAME standards, which encompasses all analyzed fatty acids. Five-point calibration curves were created from analyzing five concentration levels of the FAME standards, and the response factor for each individual fatty acid were calculated.

The GC software used is Chromeleon 7.2, which automatically performs the response factor calculation and normalization for the quantity of the internal standard C15:0ME. The formulas that the software uses are as follows:

To calculate concentration of FAME:

$$
\text { Conc.FAME }=\frac{R_{A}}{R_{I S}} \times \text { RFactor } \times \text { Conc } \cdot I S
$$

To calculate relative concentration of FAME:

$$
\% \text { Conc.FAME }=\frac{\text { Conc.FAME }}{O D W_{\text {sample }}} \times 100 \%
$$

- Conc.FAME: Concentration of specific FAME being analyzed

- $\mathrm{R}_{\mathrm{A}}$ : Response of analyte in $\mathrm{pA}$

- $\mathrm{R}_{\mathrm{IS}}$ : Response of internal standard in $\mathrm{pA}$

- RFactor: Response factor of specific FAME being analyzed

- Conc.IS: Concentration of internal standard in $\mu \mathrm{g} / \mathrm{mL}$

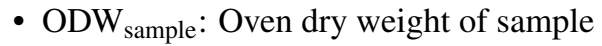

\section{Results}

\subsection{Growth and cultivation conditions}

The growth trajectories for the 32-day cultivations of Nannochloropsis oculata are shown in fig. 13. Only three data points were collected due to limited access to growing location and limited time. After 32 days of cultivation, the absorbance values obtained in the L-NP, Baseline, and H-NP nutrient concentrations measured $0.91,0.99$, and 1.18 , respectively (table 3 ). The $\mathrm{pH}$ levels and temperature were held constant between $9.63-9.91$ and $22.5-23.5^{\circ} \mathrm{C}$, respectively, throughout the cultivation period in all nutrient concentration levels.

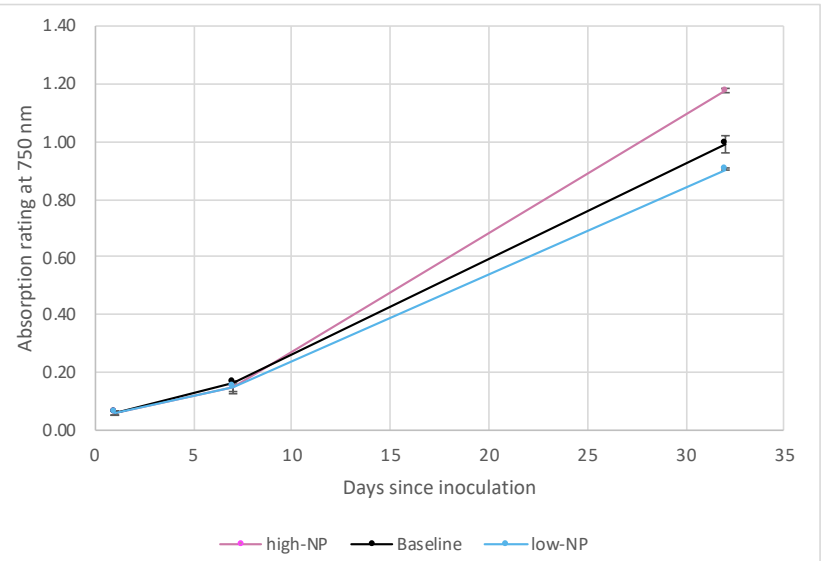

Figure 13. Growth trajectory of 32-day cultivations in H-NP, Baseline, and L-NP nutrient concentrations represented as change in absorbance over time. For each, the mean $( \pm S D)$ is given, $n=2$.

\subsection{Biomass density}

The cell density for the 32-day cultivations calculated through harvesting via flocculation of algae suspension is shown in table 4 . After 32 days of cultivation, the cell density attained in the L-NP, Baseline, and H-NP nutrient concentrations measured $0.229,0.360$, and $0.235 \mathrm{~g} / \mathrm{L}$, respectively (table 4). However, after performing a one-way ANOVA, it was found that the p-value of 0.522 is much greater than 0.05 , therefore, the data collected is not statistically significant. A possible error is explained in section 4.1.1.

\begin{tabular}{lll}
\hline L-NP & Baseline & H-NP \\
\hline $0.229(0.161)$ & $0.360(0.073)$ & $0.235(0.095)$ \\
\hline
\end{tabular}

Table 4. Biomass concentration data of 32-day cultivations in L-NP, Baseline, and H-NP nutrient concentrations. All units are in $\mathrm{g} / \mathrm{L}$. For each, the mean $( \pm S D)$ is given, $n=2, \mathrm{p}=$ 0.522 .

\subsection{Fatty acid composition}

The fatty acids that were detected and resolved to isomer level were C16:0, C16:1, C18:0, C18:1c, and C18:2 (note: Fatty acids were detected in the form of FAMEs. Here, they are represented as fatty acids since the difference in molar mass has already been accounted for). The recovery of FAMEs and the internal standard following the procedure adapted from Wychen et al. is $89.5 \pm 3.5 \%$ [42,44].

The fatty acid composition by mass is shown in table 7 . The relative fatty acid composition (table 8 ) is found by finding the ratio of the concentration of a particular fatty acid to the total fatty acid concentration. Both concentrations are in relation to concentration in the analyte and are determined without the mass of algae used during transesterification.

The dominant fatty acids present in the nutrient-stressed Nannochloropsis oculata were C16:0, C16:1, and C18:0. Further, the L-NP cultivation yielded the lowest absolute 


\begin{tabular}{l|lll}
\hline Day & Lutrient Concentration & Baseline & H-NP \\
\hline 1 & $0.06(0.007)$ & $0.06(0.007)$ & $0.06(0.007)$ \\
7 & $0.15(0.014)$ & $0.17(0.007)$ & $0.15(0.021)$ \\
32 & $0.91(0.007)$ & $0.99(0.028)$ & $1.18(0.007)$ \\
\hline
\end{tabular}

Table 3. Growth data of 32-day cultivations in L-NP, Baseline, and H-NP nutrient concentrations represented as change in absorbance over time. All units are in absorbance units. For each, the mean $( \pm$ Standard Deviation $(S D))$ is given, $n=2$.

fatty acid concentration at $11.459 \mathrm{mg} / \mathrm{g}$ biomass; however, it yielded the highest concentration of MUFAs relative to all fatty acids of $62.68 \%$. In nutrient-replete conditions, the total fatty acid concentration increased by $14.216 \%$ to $13.088 \mathrm{mg} / \mathrm{g}$ biomass, though, the relative MUFA concentration decreased by $14.56 \%$ to $48.12 \%$, while the relative SFA concentration increased by $8.79 \%$ to $38.60 \%$ and relative PUFA concentration increased by $5.77 \%$ to $13.28 \%$.

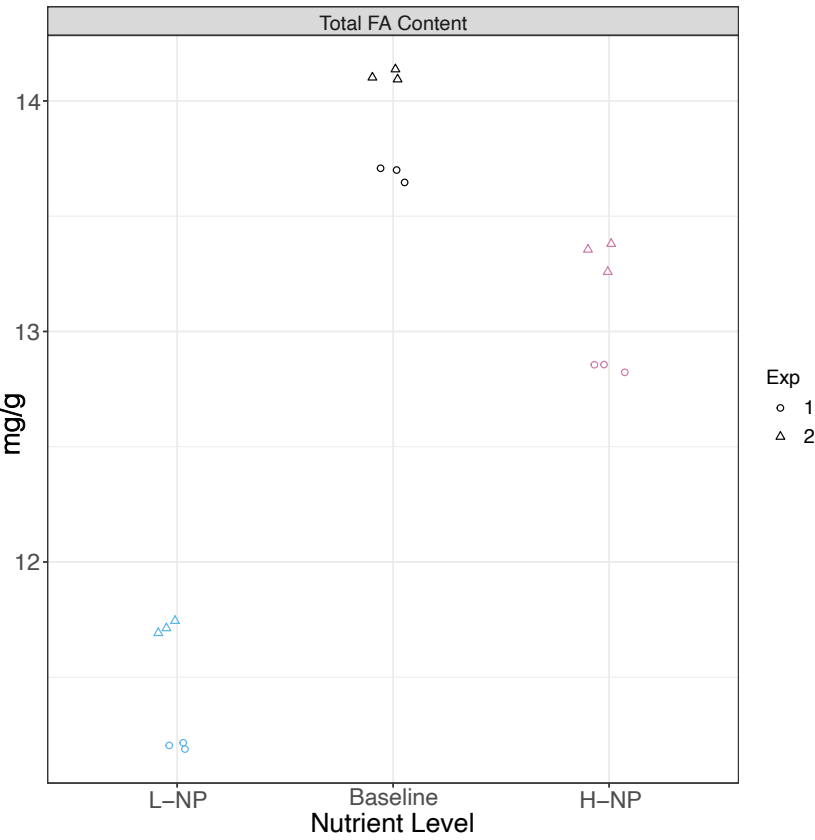

Figure 14. Total fatty acid composition showing data from all experiments and replicates. $n=2$.

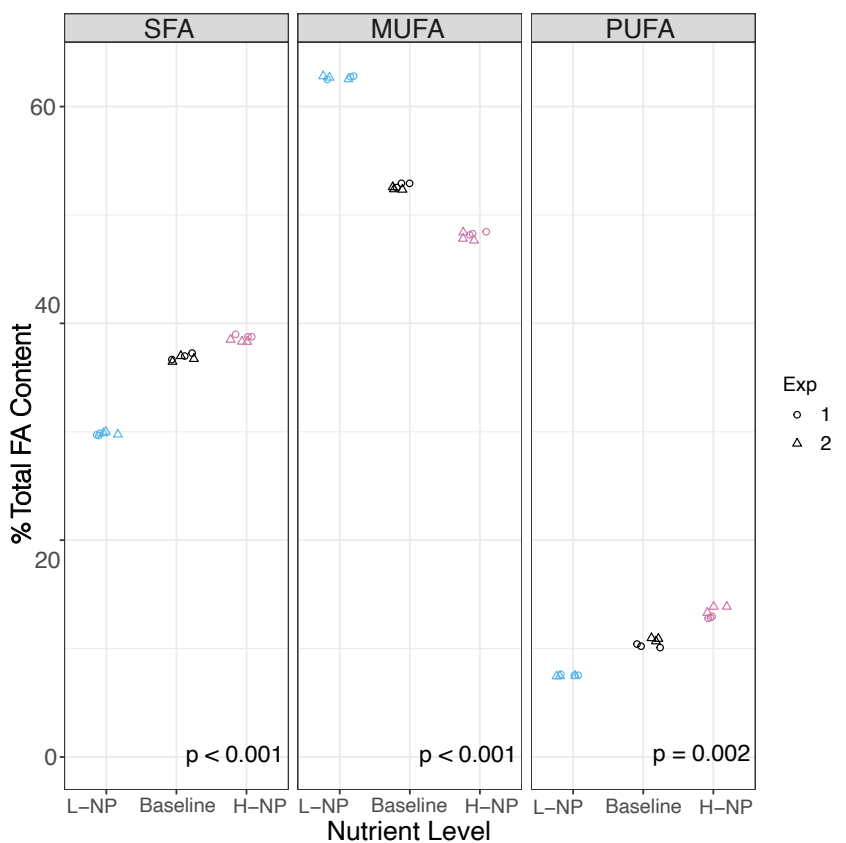

Figure 15. Relative fatty acid composition by type. $n=2$.

The data collected can be easily visualized in fig. 14 and fig. 15. Both plots include data from all experiments and all replicates. Plotting symbols represent replicates, and overlapping symbols were shifted slightly on the $\mathrm{x}$-axis for better visual representation. Replicates were averaged to obtain mean values.

Figure 14 shows the total fatty acid concentration plotted with respect to the nutrient concentration. Doubling the nutrient concentration from L-NP to Baseline represents a significant increase in the total fatty acid concentration by more than $2 \mathrm{mg}$ fatty acids per $\mathrm{g}$ of biomass.

Figure 15 shows the relative fatty acid concentration for each fatty acid type with respect to the nutrient concentration. It is seen that the L-NP cultivation produces the highest concentration of MUFA and lowest concentration of both SFA and PUFA. To confirm statistical significance, a one-way ANOVA test is performed, producing a p-value of less than 0.05 . Therefore, it is concluded that the data collected is statistically significant. 


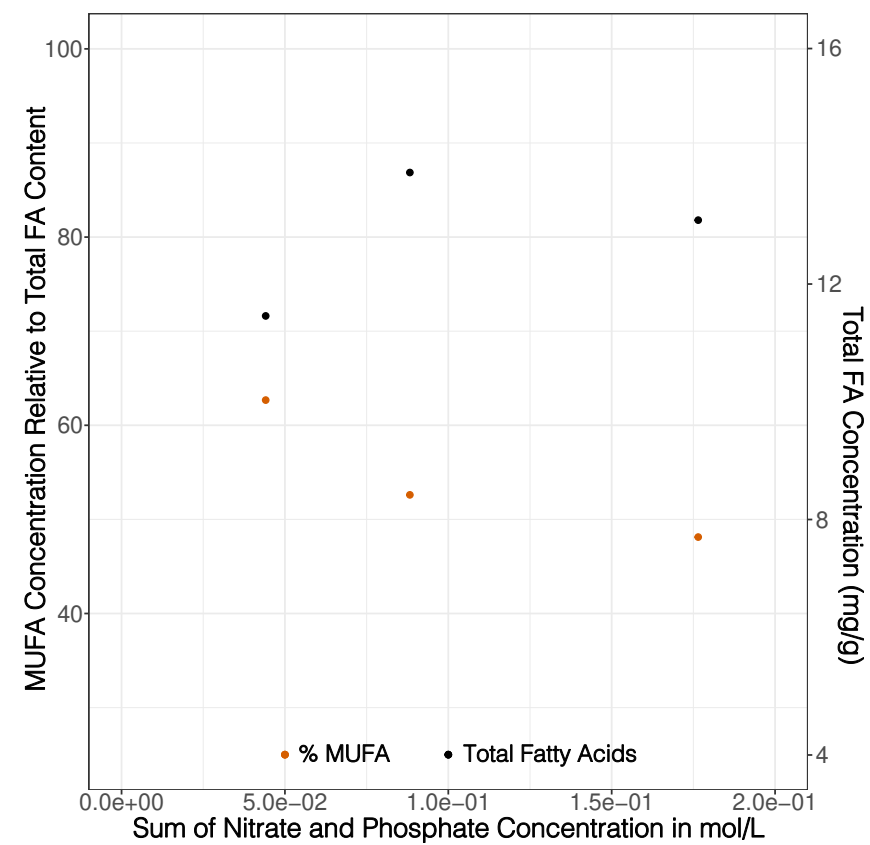

Figure 16. Change in relative MUFA content and absolute fatty acid concentration with respect to nutrient concentration cultivations in H-NP, Baseline, and L-NP nutrient concentrations.

Figure 16 shows the change in relative MUFA content and absolute fatty acid concentration with respect to the nutrient concentration. As the nutrient concentration is decreased, the relative concentration of MUFA increases; however, the total fatty acid concentration decreases. The contrary is also true: as the nutrient concentration is increased, the relative concentration of MUFA decreases while the total fatty acid conentration increases. The anomaly with the H-NP cultivation is addressed in section 4.2.

\section{Possible Inaccuracies}

\subsection{Biomass density, growth, and cultivation condi- tions}

Although this study is centered around improving the quality of biodiesel, it is still advantageous to find the effect of increasing lipid quality on the total lipids produced. To achieve this, the exact dry biomass of algae harvested must be calculated. In doing so, the algal biomass concentration was also found, which produced different results compared to that of the absorbance reading table 4 . Possible errors that caused a difference in results are explained in the following subsections.

\subsubsection{Biomass density and concentration}

To measure the exact dry biomass concentration of the algae suspensions, vacuum filtration was used to harvest the algae after flocculation. However, during the filtration, it was noticed that the filtrate is slightly green when filtering all samples. The filtrate of $\mathrm{H} 1$ and $\mathrm{H} 2$ were slightly darker in color than B1 and B2 (see fig. 10). This phenomenon can be explained by inaccuracies during the flocculation and filtration processes.

The H-NP cultivation has a higher biomass density than the baseline cultivation given its higher absorbance measurement (table 3) at the time of harvesting. Since there is increased biomass in H-NP, more flocculant must be used to flocculate all algae. However, the same volume of flocculant was used across all algae suspensions. This means that the same mass of algae was flocculated.

Further, past research has shown that algae cell sizes are smaller in higher nutrient concentrations than in lower nutrient concentrations when grown for the same period [45].

The combined effect of an overall smaller algae cell size, a lower percentage of algae flocculated, and inefficient filter paper pore size caused the inaccuracies seen in table 4 .

Although there might be a possible inaccuracy, it does not impact any other data collected in this study since no data relied on the measurement of biomass concentration in the cultivation suspension.

\subsection{Possible inaccuracies in oven dry weight}

Due to the flocculation mechanism and process, excess $\mathrm{Al}^{3+}$ ions may be stuck to the algae cells, causing inaccuracies when finding the oven dry weight and creating a mass reading higher than in reality.

To mitigate this possible inaccuracy, further experimentation is required to measure mass of $\mathrm{Al}^{3+}$ ions stuck to the algae by finding the mass of the evaporated filtrate. However, this involves using a finer filter paper and accounting for the mass of the nutrients and trace metals in the growing media.

The overall implication of a higher than in reality oven dry weight is skewing the absolute fatty acid content lower than actual values. This effect is more prominent in algae grown in $\mathrm{H}-\mathrm{NP}$ due to an overall smaller cell size. However, this does not impact the validity of this study since the quality of the biodiesel produced is based on the relative concentration of fatty acids, which does not involve the dry weight of algae used. Further, this does not impact the effect of changing nutrient composition on the relative quantity of fatty acids produced.

\subsection{Possible gas chromatography analysis inaccu- racies}

GC is regarded as the "Golden Standard" for FAME and fatty acid analysis; however, there are still possible inaccuracies specific to this study. Since the GC column used in this study was not a dedicated FAME column, it produced different results than dedicated FAME columns. For example, the observed elution order of $\mathrm{C} 16: 0 \mathrm{ME}$ and $\mathrm{C} 16: 1 \mathrm{ME}$ is reversed compared to chromatograms produced by dedicated FAME columns. This is due to the low-polarity of the TG5MS column used compared to the high-polarity of dedicated columns.

The elution order of a molecule is determined by a few aspects, including: (1) the molar mass of the molecule (a 
higher molar mass would increase elution time) and (2) the interaction between the molecule and the stationary phase of the column (greater interaction would increase elution time) [46]. C16:0ME has a slightly higher molar mass than C16:1ME, by $2.1 \mathrm{~g} / \mathrm{mol}$. However, C16:0ME interacts relatively less with the stationary phase than $\mathrm{C} 16: 1 \mathrm{ME}$ when compared to the interactions of dedicated FAME columns. This is due to the fact that $\mathrm{C} 16: 1 \mathrm{ME}$ is more polar than $\mathrm{C} 16: 0 \mathrm{ME}$ [47]; therefore, C16:1ME would interact relatively more than C16:0ME in a lower polarity column such as the one used in this study [48].

Although the elution order of C16:0ME and C16:1ME is found to be reversed compared to dedicated FAME columns, this does not affect the data collected since the same concentration of analyte is eluted and measured. The difference in order merely causes a difference in retention time reading, which is not considered during gas chromatography quantification other than to identify the molecule that is eluted.

\section{Discussion}

\subsection{Nannochloropsis oculata produces increased relative MUFA concentrations in nutrient- stressed conditions}

It is found that stressing algae during growth through nutrient starvation produces a significantly higher monounsaturated fatty acid content (MUFA) than nutrient-replete conditions (four times higher concentration) by $14.56 \%$ (fig. 15 and table 8). Further, data shows that a decrease in nutrient concentration by four times from the H-NP cultivation to the L-NP cultivation decreases both the concentration of saturated fatty acids (SFA) and polyunsaturated fatty acids (PUFA) by $8.79 \%$ and $5.77 \%$, respectively (table 8 ). This finding strongly supports the hypothesis. Therefore, the hypothesis that the Nannochloropsis grown in low nitrate and low phosphate concentrations would yield a higher MUFA percentage and lower SFA and PUFA percentage is accepted. By stressing the algae, the fatty acid profile becomes closer to that of canola oil than that of soybean oil. Therefore, the research goal is met.

\subsection{Nannochloropsis oculata produces decreased total fatty acids in nutrient-stressed conditions}

The absolute concentration of lipids decreases as the nutrient concentration decreases (fig. 14), which corroborates previous research data [49]. This supports the second half of the hypothesis that the Nannochloropsis grown in low nitrate and low phosphate concentrations would produce a relatively lower lipid concentration. Therefore, the second half of the hypothesis is also accepted.

\subsection{Theory supported by data}

Data shown in table 8 supports the theory behind the hypothesis (seen in section 1.5 that an increased lipid concentration would result in less overall desaturation and elongation. The data supports this theory since the relative concentration of MUFAs in L-NP is $30.26 \%$ more than in H-NP; therefore, more fatty acids are desaturated only one time to form MUFAs. Further, the relative concentration of longer 18-carbon chain fatty acids is $10.76 \%$ less in L-NP than in H-NP; therefore, less fatty acids are elongated. Thus, the data supports the theory that desaturation and elongation are overall correlated.

\subsection{Practical significance}

The data collected is statistically significant (fig. 15); however, this does not prove practical significance, which is one of the most important subjects to consider.

Comparing the fatty acid composition of the L-NP cultivation to soybean oil and canola oil in table 5, the MUFA concentration of L-NP is close to that of canola oil and much higher than that of soybean oil, which is an indicator that the quality of L-NP biodiesel is comparable to that of canola oil and much higher than that of soybean oil. The SFA and PUFA concentrations are nearly entirely opposite of each other in L-NP and canola oil, which affects the cetane number, cloud point, and oxidative stability of the biodiesel produced.

To analyze how the altered concentration of fatty acids affects biodiesel quality, the cetane number, cloud point, and oxidative stability are used. Table 6 compares the quality of LNP-based, canola oil-based, and soybean oil-based biodiesel and petroleum-based diesel. The values of L-NP biodiesel are estimated through analysis and comparisons of individual fatty acid types with current feedstocks [50,51]. (1) The cetane number of L-NP biodiesel is approximately 62, which is significantly greater than all other cetane numbers, including that of petroleum-based diesel. This signifies that L-NP biodiesel would have the highest combustion quality in a diesel engine, thus burning more cleanly and having lesser emissions while increasing performance. (2) The cloud point is a measure of the fluidity of diesel and biodiesel fuel at a low temperature; therefore, a lower number is desirable. The cloud point of L-NP biodiesel is approximately equal to that of canola biodiesel and is comparable to that of diesel. A vital comparison is that the cloud point of L-NP biodiesel is significantly lower than that of soybean biodiesel, which asserts the fact that L-NP biodiesel will be much more practical in the industry and the number of diesel vehicles capable of burning L-NP biodiesel is substantially more than vehicles that can burn soybean biodiesel. (3) The final and crucial indicator of fuel quality is oxidative stability, a measure of the storage capabilities of the fuel. The measure of oxidative stability has units of milligrams of insoluble particles per 100 milliliters of fuel. Therefore, a lower number is desirable, which corresponds to a more pure fuel. The data for oxidative stability used in this study was collected by the National Renewable Energy Laboratory following ASTM D2274 — Standard Test Method for Oxidation Stability of Distillate Fuel Oil (Accelerated Method) [51]. D2274 measures the inherent stability of middle distillate petroleum fuel under accelerated oxidizing conditions. In the method [52], the fuel is aged at $95^{\circ} \mathrm{C}$ for 16 hours while oxygen is bubbled through the sample at a rate of $3 \mathrm{~L} / \mathrm{h}$. The sample is then cooled to room temperature 


\begin{tabular}{l|lllll}
\hline & L-NP & Baseline & H-NP & Soybean Oil & Canola Oil \\
\hline \% SFA & $29.81(0.02)$ & $36.85(0.17)$ & $38.60(0.33)$ & 16.5 & 7.6 \\
\% MUFA & $62.68(0.01)$ & $52.61(0.25)$ & $48.12(0.24)$ & 23.7 & 62.9 \\
\% PUFA & $7.51(0.08)$ & $10.54(0.43)$ & $13.28(0.57)$ & 59.7 & 30.9
\end{tabular}

Table 5. The fatty acid composition of algal lipids compared to that of soybean and canola oil [12]. For algal lipids, the mean $( \pm S D)$ is given, $n=2$.

\begin{tabular}{|c|c|c|c|c|}
\hline & L-NP Biodiesel & Canola Biodiesel & Soybean Biodiesel & Petroleum-based \\
\hline Cetane Number & $\approx 62$ & 55 & 47 & 47 \\
\hline Cloud Point $\left({ }^{\circ} \mathbf{C}\right)$ & $\approx-3.3$ & -3.3 & 0.9 & -7 \\
\hline Oxidative Stability (mg/100mL) & $<6.2$ & 44.9 & 16.0 & 2.3 \\
\hline
\end{tabular}

Table 6. Comparison between L-NP algal lipid, soybean oil, canola oil, and petroleum-based diesel qualities. Numbers are estimated through analysis and comparison with current feedstocks [50,51].

before filtering to obtain the filterable insolubles. The greater values for canola biodiesel and soybean biodiesel highlight the drawbacks of current biodiesel feedstocks since they are significantly less stable compared to diesel. Comparing LNP biodiesel to petroleum-based diesel highlights one of the greatest advantages of L-NP algal lipids in terms of quality since they have similar oxidative stability measures.

Overall, the findings prove to be practically significant in the industry and can vastly improve the quality of biodiesel produced compared to all current feedstocks. This is crucial in the mission to produce a biodiesel that all diesel engines can combust.

\subsection{Maximize practicality}

To maximize the practicality of producing algal biodiesel in an industrial setting, both the total fatty acid concentration and relative MUFA concentration must be maximized. This would maximize both quality and quantity. However, previous research has concluded that quality and quantity are not compatible, and researchers were forced to choose one or the other [49]. All previous research opted for quantity. However, this significantly reduces the applicability of biodiesel in society. Having a high quantity of lower quality fuel that cannot be used by many diesel engines is not a viable solution. This study confirmed these observations between quality and quanitity. As shown in fig. 16, opting for higher quantity corresponds to a lower MUFA concentration, resulting in a lower quality biodiesel.

In this study, both high quality and high quantity were obtained. By growing the algae at the L-NP nitrate and phosphate nutrient concentration and slightly starving the algae, an excellent quality biodiesel that is comparable to petroleumbased diesel is produced. The L-NP algal biodiesel is suitable for use in all diesel engines. Most importantly, such high quality is reached without compromising productivity when compared to all other biodiesel feedstocks. While it has a $12.447 \%$ lower productivity than Nannochloropsis grown in H-NP nutrient concentration (table 7), L-NP still has greater than 100 times the productivity of all current biodiesel feedstock (table 2).

Further, it is envisioned that in an industrial setting, algae will be grown in large pools near waste processing facilities. In this setting, there will be adequate sunlight and air to promote algal growth. As previously mentioned (section 1.3), algae is capable of filtering waste while simultaneously increasing its productivity, therefore it is twice as effective. In these conditions, algae will not only produce a high quality feedstock for biodiesel, but it will also partially eliminate the need for large waste treatment facilities through chemical processes. This will further decrease the environmental impacts of society.

\section{Conclusion}

High-quality biodiesel with MUFA content greater than $60 \%$ is crucial to the growth and development of the biodiesel industry. Thus far, all research regarding biodiesel, more specifically algal biodiesel, has been focused on producing the highest quantity of lipids and maximizing the production of biodiesel. However, having large amounts of biodiesel is of no use unless the quality of biodiesel nears that of petroleumbased diesel.

Unfortunately, guidelines on the quality of biodiesel produced from first and second-generation feedstocks (see section 1.2.3 and section 1.2.4) are not nearly strict enough to maintain high-quality biodiesel by requiring a specific concentration of MUFA in the feedstock used. The quality of biodiesel produced by soybean oil is much worse than petroleum-based diesel due to its higher viscosity and higher freezing point, thus significantly decreasing the widespread implementation of biodiesel since many engines are unable to combust low-quality biodiesel effectively. The findings in this research provide scientific statistics and evidence that would enable the guidelines and government regulations to become more strict.

Although canola oil produces the highest quality biodiesel 
out of all feedstocks used in the United States due to its highest MUFA content and lower SFA and PUFA content, it is not nearly the most used feedstock. This is due to the high demand for canola oil as a cooking oil. This drawback proves that an effective feedstock must be grown solely for the purpose of biodiesel production, not for agricultural purposes with biodiesel production as a secondary product.

A high-quality feedstock is logistically required to increase the likelihood of biodiesel fully replacing diesel as fuel used in diesel engines. Further, it is crucial that this feedstock does not compete with the agricultural industry for vast amounts of arable land. What is discovered in this research suggests that algae is the perfect candidate for this vision due to its incredibly high productivity and no requirement for arable land.

This study aimed to find the optimal nutrient concentration for Nannochloropsis growth that will both maximize the relative MUFA content of the algae, which therefore maximizes the quality of biodiesel produced, but also yields high productivity. A scientific breakthrough was made in finding that a nitrate and phosphate nutrient concentration of $4.41 \times 10^{-2} \mathrm{M}$ and $1.81 \times 10^{-5} \mathrm{M}$ should be used in the algae cultivation medium to achieve a fine balance of improving biodiesel quality while maintaining high productivity. The MUFA concentration of algae matches that of canola oil when grown in a medium at the specified nutrient concentration. Furthermore, the cetane number and oxidative stability are better than all current biodiesel feedstocks, in some cases better than even petroleum-based diesel. Most importantly, the Nannochloropsis algae continued to maintain high productivity at this concentration. These characteristics define algal biodiesel produced by algae grown in nutrient-stressed conditions as the highest quality biodiesel that is currently available and is most suitable for large-scale production.

In summary, this study has determined one of the most crucial and often overlooked aspects of biodiesel production, which is the optimization of quality, along with quantity, through determining the optimal growing conditions of Nannochloropsis oculata for high quality and quantity biodiesel production. Together, algae is a high quality, high productivity, and waste processing feedstock. Further, the algae accomplishes this without requiring arable land. These findings will prove vital to the development of algal biofuels, which are the future of the biodiesel industry.

\section{Acknowledgments}

The author would like to thank the following Shady Side Academy teachers: Dr. Devon Renock for mentoring me throughout this project, helping answer my uncountable number of questions, and, most importantly, setting up the GC and installing the new column; Dr. Joe Martens for helping purchase all of the supplies needed by this research project; Ms. Sabundayo for her continued support throughout this project; Dr. Scott Peterson for helping to supervise my early morning experiments and open countless doors. Also, thank you to the University of Pittsburgh Staff Ms. Kristine Cooper for answering statistics-related questions.

All photos are taken by Steven D. Liu. 


\section{Appendices}

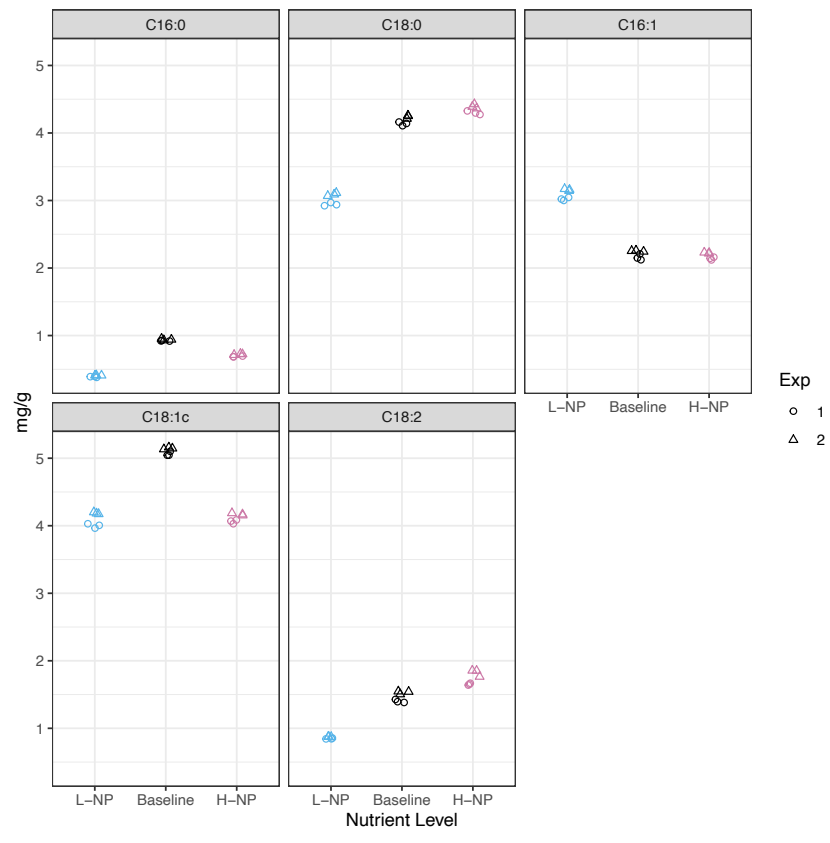

Figure 17. Fatty acid composition showing data from all experiments and replicates. $n=2$.

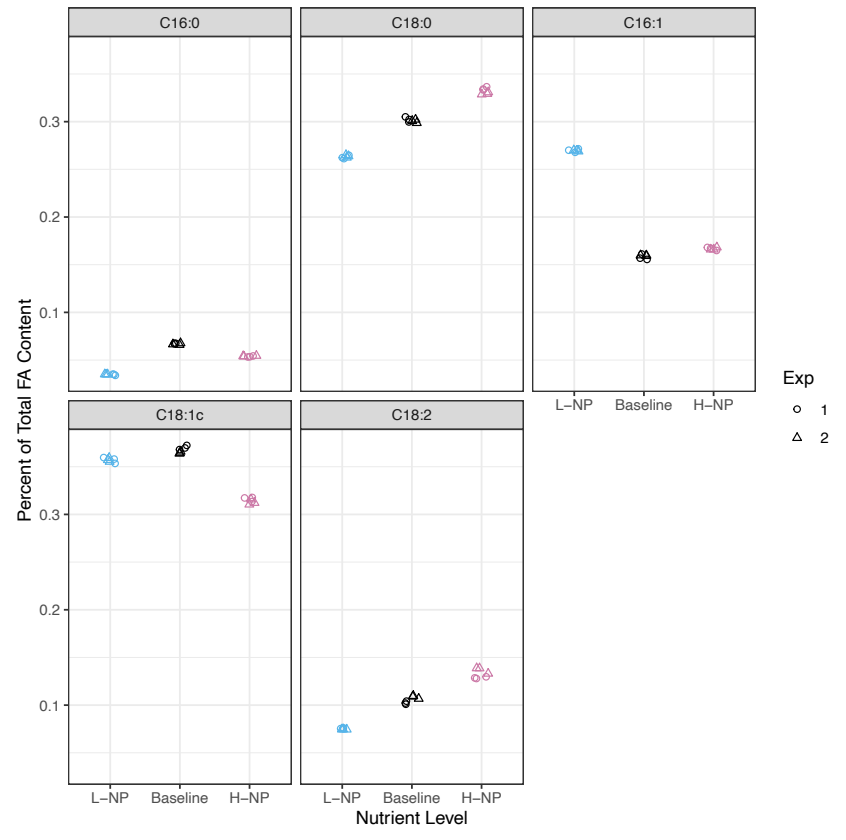

Figure 18. Relative fatty acid composition showing data from all experiments and replicates. $n=2$.

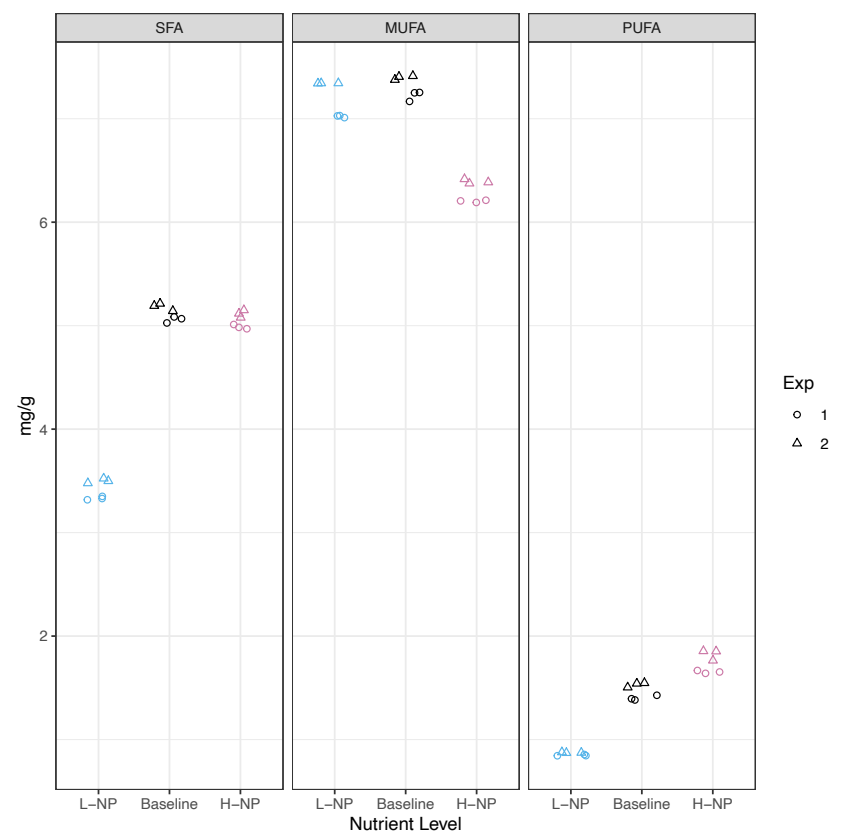

Figure 19. Fatty acid composition by type. $n=2$. 


\begin{tabular}{lccc}
\hline Nutrient Concentration & L-NP & Baseline & H-NP \\
\hline Total FA Content & 11.459 & 13.898 & 13.088 \\
\hline C16:0 & Saturated Fatty Acids (SFA) & $0.705(0.023)$ \\
C18:0 & $0.399(0.015)$ & $0.932(0.014)$ & $4.346(0.067)$ \\
$\Sigma$ SFA & $3.0166(0.104)$ & $4.189(0.073)$ & $5.052(0.090)$ \\
\hline C16:1 & $3.416(0.119)$ & $5.121(0.087)$ & $2.182(0.057)$ \\
C18:1c & Monounsaturated Fatty Acids (MUFA) & $4.116(0.077)$ \\
$\Sigma$ MUFA & $3.090(0.095)$ & $2.206(0.065)$ & $6.298(0.134)$ \\
\hline C18:2 & $4.093(0.131)$ & $5.105(0.058)$ & $1.739(0.120)$ \\
$\Sigma$ PUFA & $7.183(0.226)$ & $7.311(0.123)$ & $1.739(0.120)$ \\
\hline
\end{tabular}

Table 7. The concentration of each identified fatty acids in $\mathrm{mg} / \mathrm{g}$ oven dry weight after 32 days cultivation in L-NP, Baseline, and H-NP nutrient concentrations. For each, the mean $( \pm S D)$ is given, $n=2$.

\begin{tabular}{lccc}
\hline Nutrient Concentration & L-NP & Baseline & H-NP \\
\hline \multicolumn{4}{c}{ Saturated Fatty Acids (SFA) } \\
\hline C16:0 & $3.48 \%(0.02)$ & $6.70 \%(0.05)$ & $5.39 \%(0.03)$ \\
C18:0 & $26.32 \%(0.07)$ & $30.14 \%(0.13)$ & $33.21 \%(0.36)$ \\
\hline SFA & $29.81 \%(0.09)$ & $36.85 \%(0.17)$ & $38.60 \%(0.33)$ \\
\hline C16:1 & Monounsaturated Fatty Acids (MUFA) \\
C18:1c & $26.97 \%(0.02)$ & $15.87 \%(0.12)$ & $16.67 \%(0.01)$ \\
L MUFA & $35.71 \%(0.01)$ & $36.74 \%(0.38)$ & $31.45 \%(0.24)$ \\
\hline C18:2 & $62.68 \%(0.01)$ & $52.61 \%(0.25)$ & $48.12 \%(0.24)$ \\
\hline PUFA & Polyunsaturated Fatty Acids (PUFA) & $13.28 \%(0.57)$ \\
\hline
\end{tabular}

Table 8. The fatty acids composition relative to total fatty acid content after 32 days cultivation in L-NP, Baseline, and H-NP nutrient concentrations. For each, the mean $( \pm S D)$ is given, $n=2$. 


\section{References}

[1] BP. Statistical Review of World Energy. BP, 69th edition, 2020.

[2] Anderson Cooper. Bill Gates: How the world can avoid a climate disaster. https://www.cbsnews.com/news/ bill-gates-climate-change-disaster-60-minutes-2021-02-14/, February 2021. An interview with Bill Gates.

[3] IPCC. Climate Change 2014: Synthesis Report. Contribution of Working Groups I, II and III to the Fifth Assessment Report of the Intergovernmental Panel on Climate Change [Core Writing Team, R.K. Pachauri and L.A. Meyer (eds.)]. IPCC, 2014.

[4] Rebecca Lindsey. Climate Change: Atmospheric Carbon Dioxide. https://www. climate.gov/news-features/understanding-climate/ climate-change-atmospheric-carbon-dioxide, August 2020.

[5] DOE. Fossil. https://www.energy.gov/ science-innovation/energy-sources/fossil.

[6] Office of Transportation and Air Quality. Fast Facts: U.S. Transportation Sector Greenhouse Gas Emissions, 19902018. United States Environmental Protection Agency, June 2020.

[7] Office of Transportation and Air Quality. Smog, Soot, and Other Air Pollution from Transportation. https://www.epa.gov/ transportation-air-pollution-and-climate-change/ smog-soot-and-local-air-pollution, 2020.

[8] Office of Transportation and Air Quality. Understanding Global Warming Potentials. https:/www.epa.gov/ghgemissions/ understanding-global-warming-potentials, 2020.

[9] IRENA. Global Energy Transformation: A roadmap to 2050. International Renewable Energy Agency, Abu Dhabi, 2018.

[10] UCUSA. Cars, Trucks, Buses and Air Pollution. https://www.ucsusa.org/resources/ cars-trucks-buses-and-air-pollution, July 2018.

[11] Rosalind O'Driscoll, Marc E.J. Stettler, Nick Molden, Tim Oxley, and Helen M. ApSimon. Real world CO2 and NOx emissions from 149 Euro 5 and 6 diesel, gasoline and hybrid passenger cars. Science of The Total Environment, 621:282-290, 2018.

[12] S. Kent Hoekman, Amber Broch, Curtis Robbins, Eric Ceniceros, and Mani Natarajan. Review of biodiesel composition, properties, and specifications. Renewable and Sustainable Energy Reviews, 16(1):143-169, 2012.

[13] 110th United States Congress. "Energy Independence and Security Act of 2007" (PL 110-140, 19 Dec. 2007), 106. United States Statutes at Large, pages 1492-1801.
[14] EPA. Renewable Fuel Standard Program: Standards for 2020 and Biomass- Based Diesel Volume for 2021 and Other Changes. U.S. Environmental Protection Agency, February 2020.

[15] U.S. EIA. Monthly Biodiesel Production Report. https: //www.eia.gov/biofuels/biodiesel/production/, February 2021.

[16] U.S. DOE. Renewable Fuel Standard. https://afdc.energy. gov/laws/RFS.html.

[17] DOE. Renewable Hydrocarbon Biofuels. https://afdc. energy.gov/fuels/emerging_hydrocarbon.html, 2020.

[18] National Renewable Energy Lab. Biodiesel: Handling and Use Guidelines. Technical report, U.S. Department of Energy, Golden, CO, September 2006. A guide for handling, blending, distributing, and using biodiesel fuels and biodiesel fuel blends.

[19] Vinoth Thangarasu and R. Anand. Chapter 17 - comparative evaluation of corrosion behavior of aegle marmelos correa diesel, biodiesel, and their blends on aluminum and mild steel metals. In Abul Kalam Azad and Mohammad Rasul, editors, Advanced Biofuels, Woodhead Publishing Series in Energy, pages 443-471. Woodhead Publishing, 2019.

[20] FAO. The state of food and agriculture 2008. Food and Agriculture Organization, New York, 2008.

[21] FAO. Sustainable bioenergy: a framework for decision makers. United Nations Energy, 2007.

[22] Didem Özçimen and Benan İnan. An overview of bioethanol production from algae. In Krzysztof Biernat, editor, Biofuels, chapter 7. IntechOpen, Rijeka, 2015.

[23] Digambar Singh, Dilip Sharma, S.L. Soni, Sumit Sharma, Pushpendra Kumar Sharma, and Amit Jhalani. A review on feedstocks, production processes, and yield for different generations of biodiesel. Fuel, 262:116553, 2020.

[24] U.S. EIA. Biodiesels produced from certain feedstocks have distinct properties from petroleum diesel. https: //www.eia.gov/todayinenergy/detail.php?id=36052, May 2019.

[25] General Microbiology at Boundless. Lipid molecules. https://bio.libretexts.org/Bookshelves/Introductory_ and_General_Biology/Book\%3A_General_Biology_ (Boundless)/3\%3A_Biological_Macromolecules/3.2\% 3A_Lipid_Molecules/3.2A\%3A_Lipid_Molecules, Dec 2020.

[26] Sergii Boichenko, Anna Yakovlieva, Oksana Vovk, Margaryta Radomska, Larysa Cherniak, and Irina Shkilniuk. Fundamentals of chemmotology. National Aviation University, 012019.

[27] S.C. Bhatia. 16 - biofuels: A review. In S.C. Bhatia, editor, Advanced Renewable Energy Systems, pages 403425. Woodhead Publishing India, 2014. 
[28] Muhammad Aminul Islam, Godwin A. Ayoko, Richard Brown, Doug Stuart, and Kirsten Heimann. Influence of fatty acid structure on fuel properties of algae derived biodiesel. Procedia Engineering, 56:591-596, 2013. 5th BSME International Conference on Thermal Engineering.

[29] Sukumar Puhan, N. Saravanan, G. Nagarajan, and N. Vedaraman. Effect of biodiesel unsaturated fatty acid on combustion characteristics of a di compression ignition engine. Biomass and Bioenergy, 34(8):1079-1088, 2010.

[30] U.S. EIA. Biofuels explained: Biomass-based diesel fuels. https://www.eia.gov/energyexplained/biofuels/ biodiesel.php, June 2020.

[31] Rojan P. John, G.S. Anisha, K. Madhavan Nampoothiri, and Ashok Pandey. Micro and macroalgal biomass: A renewable source for bioethanol. Bioresource Technology, 102(1):186-193, 2011. Special Issue: Biofuels - II: Algal Biofuels and Microbial Fuel Cells.

[32] Poonam Singh Nigam and Anoop Singh. Production of liquid biofuels from renewable resources. Progress in Energy and Combustion Science, 37(1):52-68, 2011.

[33] Maurycy Daroch, Shu Geng, and Guangyi Wang. Recent advances in liquid biofuel production from algal feedstocks. Applied Energy, 102:1371-1381, 2013. Special Issue on Advances in sustainable biofuel production and use - XIX International Symposium on Alcohol Fuels ISAF.

[34] Liam Brennan and Philip Owende. Biofuels from microalgae-a review of technologies for production, processing, and extractions of biofuels and co-products. $R e$ newable and Sustainable Energy Reviews, 14(2):557-577, 2010.

[35] Y.M. Sani, W.M.A.W. Daud, and A.R. Abdul Aziz. Biodiesel feedstock and production technologies: Successes, challenges and prospects. In Zhen Fang, editor, Biodiesel, chapter 4. IntechOpen, Rijeka, 2012.

[36] S.P. Singh and Priyanka Singh. Effect of co2 concentration on algal growth: A review. Renewable and Sustainable Energy Reviews, 38:172-179, 2014.

[37] Jarunan Pratoomyot, Srivilas Piyawan, and Thidarat Noiraksar. Fatty acids composition of 10 microalgal species. Songklanakarin Journal of Science and Technology, 27, 112005.

[38] Matthew P. Huber. Algae Culturing Manual v2. Algae Research Supply, 2018.

[39] Albert J. Dijkstra, Richard J. Hamilton, and Wolf Hamm. Fatty Acid Biosynthesis. In Trans Fatty Acids, chapter 1.4. Blackwell Pub, Oxford, January 2008.

[40] R.R.L. Guillard. Culture of Phytoplankton for Feeding Marine Invertebrates In: Smith W.L., Chanley M.H. (eds). Culture of Marine Invertebrate Animals, 1975.
[41] Tawan Chatsungnoen and Yusuf Chisti. Harvesting microalgae by flocculation-sedimentation. Algal Research, 13:271-283, 2016.

[42] Lieve M. L. Laurens, Matthew Quinn, Stefanie Van Wychen, David W. Templeton, and Edward J. Wolfrum. Accurate and reliable quantification of total microalgal fuel potential as fatty acid methyl esters by in situ transesterification. Analytical and Bioanalytical Chemistry, 403(1):167-178, Apr 2012.

[43] Restek. High-Resolution GC Analyses of Fatty Acid Methyl Esters (FAMEs). https://www. restek.com/Technical-Resources/Technical-Library/ Foods-Flavors-Fragrances/fff_FFAR2931A-UNV, 2018.

[44] Stefanie Van Wychen, Kelsey Ramirez, and Lieve M.L. Laurens. Determination of total lipids as fatty acid methyl esters (fame) by in situ transesterification. National Renewable Energy Laboratory (NREL), 2015.

[45] Chris J. Hulatt, René H. Wijffels, Sylvie Bolla, and Viswanath Kiron. Production of fatty acids and protein by nannochloropsis in flat-plate photobioreactors. PLOS ONE, 12(1):1-17, January 2017.

[46] Jean-Yves de Saint Laumer, Esmeralda Cicchetti, Philippe Merle, Jonathan Egger, and Alain Chaintreau. Quantification in Gas Chromatography: Prediction of Flame Ionization Detector Response Factors from Combustion Enthalpies and Molecular Structures. Analytical Chemistry, 82(15):6457-6462, Aug 2010.

[47] H. S. Murali, M. S. Mohan, K. S. Manja, and R. Sankaran. Polar and nonpolar lipids and their fatty acid composition of a fewfusarium species. Journal of the American Oil Chemists' Society, 70(10):1039-1041, Oct 1993.

[48] S. Lipsky and R. Landowne. Evaluation of a stationary phase for fatty acid analysis by gas-liquid chromatography. Annals of the New York Academy of Sciences, 72:666 - 674, 122006.

[49] Kjell Reitan, Jose Rainuzzo, and Yngvar Olsen. Effect of nutrient limitation on fatty acid and lipid content of marine microalgae. Journal of Phycology, 30:972 - 979, 102004.

[50] Zulqarnain, Muhammad Ayoub, Mohd Hizami Mohd Yusoff, Muhammad Hamza Nazir, Imtisal Zahid, Mariam Ameen, Farooq Sher, Dita Floresyona, and Eduardus Budi Nursanto. A comprehensive review on oil extraction and biodiesel production technologies. Sustainability, 13(2), 2021.

[51] J.A. Kinast. Production of Biodiesels from Multiple Feedstocks and Properties of Biodiesels and Biodiesel/Diesel Blends. Technical report, Gas Technology Institute, 2003.

[52] S.R. Westbrook. An Evaluation and Comparison of Test Methods to Measure the Oxidation Stability of Neat Biodiesel. Technical report, Southwest Research Institute, 2005. 